\title{
Cooperative Linear-Quadratic Mean Field Control and Hamiltonian Matrix Analysis
}

\author{
by \\ Xiang Chen \\ A thesis submitted to the Faculty of Graduate and Postdoctoral Affairs \\ in Partial Fulfillment of the requirements for the Degree of
}

\author{
Master of Science \\ in \\ Probability and Statistics \\ Carleton University \\ Ottawa, Ontario, Canada
}

(C) 2017, Xiang Chen 


\section{Abstract}

In this thesis, we present a new methodology to investigate the existence and uniqueness of the solution of the Social Certainty Equivalence (SCE) equation system related to social optima in mean field linear-quadratic-Gaussian (LQG) control problems. The methodology involves Hamiltonian matrices and continuous-time algebraic Riccati equations (CARE), and it avoids the restrictive contractive assumption typically used in a fixed point approach. For computing the stabilizing solution of CARE, we also develop a computational method related to generalized eigenvectors and Schur vectors. We further extend our method to solve a linear-quadratic mean field game.

Keywords: SCE Equation System; Hamiltonian Matrices; Continuous-time Algebraic Riccati Equations 


\section{Dedication}

I dedicate this thesis to my parents, Tianxiong Chen and Hui Li, who always encourage me and support me.

I would like to thank my supervisor, Minyi Huang, for his patient guidance and advice throughout my time as his student. 


\section{Table of Contents}

Abstract $\quad$ ii

Dedication $\quad$ iii

Table of Contents $\quad$ iv

List of Figures $\quad$ v

1 Introduction 1

1.1 Claim of Original Contributions . . . . . . . . . . . . 4

2 Social Optimization Problem 5

2.1 Description of Problem . . . . . . . . . . . . . . . 5

2.2 Eigenvalues and Eigenvectors of Hamiltonian Matrices . . . . . . . . 6

2.3 Continuous-time Algebraic Riccati Equations . . . . . . . . . . . . 8

2.4 Frequency Domain Condition . . . . . . . . . . . . . . . . 13

2.5 Existence and Uniqueness of A Solution . . . . . . . . . . . . . 15

2.6 Computational Methods . . . . . . . . . . . . . . . . . . . . . . 21

2.6 .1 The Stabilizing Solution . . . . . . . . . . . . . . . . 21

2.6 .2 Computational Results . . . . . . . . . . . . . . . 29

2.6 .3 Comparison . . . . . . . . . . . . . . . . . 35

3 Mean Field Game Problem $\quad 38$

3.1 Description of Problem . . . . . . . . . . . . . . . . . . . . . 38

3.2 Existence of A Solution . . . . . . . . . . . . . . . . 39

$\begin{array}{llr}4 & \text { Conclusion } & 43\end{array}$

$\begin{array}{ll}\text { Appendix A Matlab Code } & 47\end{array}$ 


\section{List of Figures}

Figure $2.1 \quad$ Solution when $\gamma=1 \ldots \ldots \ldots$. . . . . . . . . . . . . 32

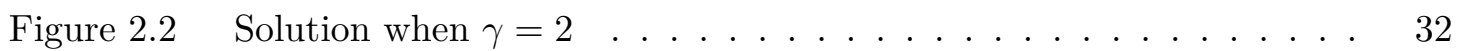

Figure $2.3 \quad$ Solution when $\gamma=3 \ldots \ldots \ldots \ldots \ldots$

Figure 2.4 Solution when $\gamma=2 \ldots \ldots \ldots \ldots \ldots \ldots$

Figure $2.5 \quad$ Solution when $\gamma=1 \ldots \ldots \ldots \ldots \ldots$

Figure $3.1 \quad$ Solution of Example $3.2 .2 \ldots \ldots \ldots \ldots$. . . . . . . . . . 42 


\section{Chapter 1}

\section{Introduction}

Mean field game (MFG) theory studies stochastic decision making problems involving a large number of noncooperative agents. In a mean field game, the individual behavior has insignificant influence on others, while the mass behavior has significant impacts on individuals. Traditional approaches to mean field optimization problems involve high complexity for analysis and computation because of the large size of the population of players. MFG theory provides a powerful methodology to reduce the complexity by applying mean field approximations, which can construct a decentralized strategy and then show an $\epsilon$-Nash equilibrium property [6]. Due to the advantages of MFG theory, it has been widely used in many domains related to large-scale dynamical systems, such as social science $[2,3]$, economics [5] and engineering [14].

There is a parallel development in social optima. Different from MFG, mean field socially optimal control problems involve a large number of cooperative agents. The aim of this model is seeking socially optimal decisions. Similar to MFG, one may consider centralized and decentralized strategies in social optima. For centralized strategies, each agent has access to information of all agents. Centralized strategies can be found by solving a standard LQG control problem [8]. For decentralized strategies, each agent only uses local information, and this is a highly desirable feature for reducing complexity. We describe a uniform agent example of social optima in mean field LQG control to show how to find decentralized strategies by mean approximations. This example is based on [8]. Throughout this thesis, we will use the same notations as in the following example. 
Consider an LQG control system of $N$ agents. The dynamics of agent $i$ are given by the stochastic differential equation (SDE):

$$
d x_{i}=A x_{i} d t+B u_{i} d t+D d W_{i}, \quad t \geq 0
$$

We use $\left(\Omega, \mathcal{F},\left\{\mathcal{F}_{t}\right\}_{t \geq 0}, P\right)$ to denote an underlying filtered probability space. The state $x_{i}$ and the control $u_{i}$ are $n$ and $n_{1}$ dimensional vectors respectively. The initial states $\left\{x_{i}(0), 1 \leq i \leq N\right\}$ are independent. The noise processes $\left\{W_{i}, 1 \leq i \leq N\right\}$ are $n_{2}$ dimensional independent standard Brownian motions adapted to $\mathcal{F}_{t}$, which are also independent of $\left\{x_{j}(0), 1 \leq j \leq N\right\}$. The constant matrices $A, B$ and $D$ have compatible dimensions. Given a matrix $Q$, the quadratic form $z^{T} Q z$ may be denoted as $|z|_{Q}^{2}$.

The individual cost for agent $i$ is given by

$$
J_{i}(u(\cdot))=E \int_{0}^{\infty} e^{-\rho t}\left[\left|x_{i}-\Phi\left(x^{(N)}\right)\right|_{Q}^{2}+u_{i}^{T} R u_{i}\right] d t
$$

where $\Phi\left(x^{(N)}\right)=\Gamma x^{(N)}+\eta$ and $x^{(N)}:=(1 / N) \sum x_{i}$ is called the mean field term. The constant matrices or vectors $\Gamma, Q \geq 0, R>0$ and $\eta$ have compatible dimensions, and $\rho>0$ is a discount factor.

The objective of the social optimization problem is to minimize the social cost, which is defined as

$$
J_{\text {soc }}^{(N)}(u(\cdot))=\sum_{i=1}^{N} J_{i}(u(\cdot)) .
$$

Denote $u_{-i}:=\left(u_{1}, \cdots, u_{i-1}, u_{i+1}, \cdots, u_{N}\right)$, and $\hat{u}^{c}=\left(\hat{u}_{1}^{c}, \cdots, \hat{u}_{N}^{c}\right)$ is the solution with centralized strategies.

Firstly, considering a new social cost $J_{i}^{0}\left(u_{i}\right)=J_{\text {soc }}^{(N)}\left(\hat{u}_{1}^{c}, \cdots, \hat{u}_{i-1}^{c}, u_{i}, \hat{u}_{i+1}^{c}, \cdots, \hat{u}_{N}^{c}\right)$, it is known that for minimizing $J_{i}^{0}$, the best strategy is $\hat{u}_{i}=\hat{u}_{i}^{c}$. This property is called person-by-person optimality [8].

Secondly, since $J_{i}^{0}$ is only optimized by $u_{i}$, terms within $J_{i}^{0}$ not affected by $u_{i}$ can be dropped. Thus, minimizing $J_{i}^{0}$ is equivalent to minimizing:

$$
J_{i}^{1}\left(u_{i}\right)=E \int_{0}^{\infty} e^{-\rho t} L\left(x_{i}, \hat{x}_{-i}^{(N)}, u_{i}\right)(t) d t
$$


where $\hat{x}_{-i}^{(N)}=(1 / N) \sum_{j \neq i} \hat{x}_{j}^{c} ; \hat{x}_{j}^{c}$ corresponds to $\hat{u}_{j}^{c}$ and

$$
\begin{aligned}
L & =x_{i}^{T}\left[\left(I-\frac{\Gamma}{N}\right)^{T} Q\left(I-\frac{\Gamma}{N}\right)+\frac{\left.(N-1) \Gamma^{T} Q \Gamma\right] x_{i}}{N^{2}}\right] \\
& -2\left(\Gamma \hat{x}_{-i}^{(N)}+\eta\right)^{T} Q\left(I-\frac{\Gamma}{N}\right) x_{i} \\
& -2\left\{\left[1-\left(1-\frac{1}{N}\right) \Gamma\right] \hat{x}_{-i}^{(N)}-\left(1-\frac{1}{N}\right) \eta\right\}^{T} Q \Gamma x_{i} \\
& +u_{i}^{T} R u_{i} .
\end{aligned}
$$

The detail of proof is in [8].

Finally, for large $N$, it is reasonable to use a deterministic function $\bar{x}$ to approximate $\hat{x}_{-i}^{(N)}$ in (1.5). Thus, the limiting optimal control problem is:

$$
\begin{aligned}
& d x_{i}=A x_{i} d t+B u_{i} d t+D d W_{i}, \quad t \geq 0 . \\
& \min J_{i}^{*}\left(u_{i}\right)=E \int_{0}^{\infty} e^{-\rho t} L^{*}\left(x_{i}, \bar{x}, u_{i}\right)(t) d t
\end{aligned}
$$

where $L^{*}\left(x_{i}, \bar{x}, u_{i}\right)(t)$ is the approximation of (1.5) and takes the form:

$$
L^{*}\left(x_{i}, \bar{x}, u_{i}\right)(t)=x_{i}^{T} Q x_{i}-2(\Gamma \bar{x}+\eta)^{T} Q x_{i}-2[(I-\Gamma) \bar{x}-\eta]^{T} Q \Gamma x_{i}+u_{i}^{T} R u_{i} .
$$

By this mean field approximation, the social cost in (1.6) only depends on $x_{i}$ and $u_{i}$. Thus, the resulting solution $\hat{u}_{i}$ only uses local information, which means this $\hat{u}_{i}$ is a decentralized strategy. On the other hand, according to LQG theory, the optimal control is:

$$
\hat{u}_{i}=-R^{-1} B^{T}\left(\Pi x_{i}+s\right),
$$

where $\Pi$ is the solution of the following algebraic Riccati equation (ARE):

$$
\rho \Pi=\Pi A+A^{T} \Pi-\Pi B R^{-1} B^{T} \Pi+Q,
$$

and $\bar{x}$ and $s$ satisfy the Social Certainty Equivalence(SCE) equation system:

$$
\begin{aligned}
& \frac{d \bar{x}}{d t}=\left(A-B R^{-1} B^{T} \Pi\right) \bar{x}-B R^{-1} B^{T} s \\
& \frac{d s}{d t}=Q_{\Gamma} \bar{x}+\left(\rho I-A^{T}+\Pi B R^{-1} B^{T}\right) s+\eta_{\Gamma},
\end{aligned}
$$


where $\bar{x}(0)=x_{0},(\bar{x}, s) \in C_{\rho / 2}\left([0, \infty), \mathbb{R}^{2 n}\right)$ (see Section 2.1 for the definition), $Q_{\Gamma}=$ $\Gamma^{T} Q+Q \Gamma-\Gamma^{T} Q \Gamma$ and $\eta_{\Gamma}=\left(I-\Gamma^{T}\right) Q \eta$.

Now, the social optimization problem has been reduced to solving a SCE equation system [8]. Thus, if there exists a solution $(\bar{x}, s)$, a decentralized control $\hat{u}_{i}$ can be found by (1.7). To summarize, by person-by-person optimality and mean field approximations, the solution of the social optimization problem is equivalent to solving the ODE system (1.9)(1.10). Thus, the key issue here is whether there exists a unique solution of $(\bar{x}, s)$. When

$Q_{\Gamma}$ is negative semidefinite, existence and uniqueness of the solution has been proved [8, Theorem 4.3].

There are some similar social optimization problems presented in [1], [10] and [19]. In [10], the authors introduce a mean field LQG game model involving a major player. Here the major player has a strong impact on other players. Another related work is [1], which considers nonlinear mean field optimal control to minimize a social cost.

In this thesis, we develop a new approach for the social optimization problem to analyze the solution of SCE equation system (1.9)-(1.10) when $Q_{\Gamma}$ is only symmetric. Our method involves Hamiltonian matrices, frequency domain conditions and continuous-time algebraic Riccati equations (CARE).

The organization of this thesis is as follow. Chapter 2 develops a new methodology to solve the SCE equation system and proves the existence and uniqueness of a solution $(\bar{x}, s)$ in $C_{\rho / 2}\left([0, \infty), \mathbb{R}^{2 n}\right)$ by identifying a unique initial condition $s_{0}$. The mean field game solution is presented in Chapter 3. Chapter 4 concludes the thesis.

\subsection{Claim of Original Contributions}

We prove existence and uniqueness of a solution to SCE equation systems by using a decomposition technique based on Hamiltonian matrix analysis and develop the computational method to solve a CARE when a related state weight matrix is indefinite. We further extend this approach to solve a mean field game. 


\section{Chapter 2}

\section{Social Optimization Problem}

\subsection{Description of Problem}

In Chapter 1, we have described a social optimum problem in mean field LQG control to find decentralized strategies by person-by-person optimality and mean field approximations.

Definition 2.1.1. For $N$ is an integer and $r$ is a positive real number, define $C_{r}\left([0, \infty), \mathbb{R}^{N}\right)$ as a set including all function $f \in C\left([0, \infty), \mathbb{R}^{N}\right)$ such that $\sup _{t \geq 0}|f(t)| e^{-r^{\prime} t}<+\infty$, for some positive real number $r^{\prime}<r$. Here, $r^{\prime}$ may depend on $f(t)$.

Now our interest is to analyze the solution of the following ODE system:

$$
\begin{aligned}
& \frac{d \bar{x}}{d t}=\left(A-B R^{-1} B^{T} \Pi\right) \bar{x}-B R^{-1} B^{T} s \\
& \frac{d s}{d t}=Q_{\Gamma} \bar{x}+\left(\rho I-A^{T}+\Pi B R^{-1} B^{T}\right) s+\eta_{\Gamma},
\end{aligned}
$$

where $\bar{x}(0)=x_{0}$, both $\bar{x}$ and $s$ are in $C_{\rho / 2}\left([0, \infty), \mathbb{R}^{n}\right), Q_{\Gamma}=\Gamma^{T} Q+Q \Gamma-\Gamma^{T} Q \Gamma$ and $\eta_{\Gamma}=\left(I-\Gamma^{T}\right) Q \eta$. Since the initial condition $s_{0}$ is unspecified, a key part of finding a solution is to determine the initial condition $s_{0}$.

We can transform the coefficient matrix in the ODE system (2.1)-(2.2) to a Hamiltonian matrix by the following transformation:

$$
\tilde{x}=e^{-\rho t / 2} \bar{x}, \quad \tilde{s}=e^{-\rho t / 2} s,
$$


and then we obtain new differential equations for $(\tilde{x}, \tilde{s})$ :

$$
\left(\begin{array}{c}
\frac{d \tilde{x}}{d t} \\
\frac{d \tilde{s}}{d t}
\end{array}\right)=\underbrace{\left(\begin{array}{cc}
A-B R^{-1} B^{T} \Pi-\frac{\rho}{2} I & -B R^{-1} B^{T} \\
Q_{\Gamma} & \frac{\rho}{2} I-A^{T}+\Pi B R^{-1} B^{T}
\end{array}\right)}_{H}\left(\begin{array}{c}
\tilde{x} \\
\tilde{s}
\end{array}\right)+\left(\begin{array}{c}
0 \\
\tilde{\eta}_{\Gamma}
\end{array}\right),
$$

where $\tilde{x}(0)=x_{0}$ and $\tilde{\eta}_{\Gamma}=e^{-\frac{\rho}{2} t} \eta_{\Gamma}$.

Denote $\mathcal{A}=A-B R^{-1} B^{T} \Pi-\frac{\rho}{2} I$ and

$$
H=\left(\begin{array}{cc}
\mathcal{A} & -B R^{-1} B^{T} \\
Q_{\Gamma} & -\mathcal{A}^{T}
\end{array}\right)
$$

Then

$$
\left(\begin{array}{c}
\frac{d \tilde{x}}{d t} \\
\frac{d \tilde{s}}{d t}
\end{array}\right)=H\left(\begin{array}{c}
\tilde{x} \\
\tilde{s}
\end{array}\right)+\left(\begin{array}{c}
0 \\
\tilde{\eta}_{\Gamma}
\end{array}\right)
$$

We should notice that in $\operatorname{ODE}(2.2), \eta_{\Gamma}$ is a constant vector. However, in system $(2.3), \tilde{\eta}_{\Gamma}$ is a function of $t$. Indeed, since $\rho>0, \lim _{t \rightarrow+\infty} \tilde{\eta}_{\Gamma}=0$, which means that it does not result in much difficulty in finding a bounded solution of ODE (2.3).

\subsection{Eigenvalues and Eigenvectors of Hamiltonian Matrices}

Before analyzing the solution of ODE system (2.3), we introduce a special class of matrices - Hamiltonian matrices.

Definition 2.2.1. A matrix $K \in \mathbb{R}^{2 n \times 2 n}$ is called a Hamiltonian matrix if $J K$ is symmetric, where

$$
J=\left(\begin{array}{cc}
O_{n} & I_{n} \\
-I_{n} & O_{n}
\end{array}\right)
$$

Since $J H=\left(\begin{array}{cc}Q_{\Gamma} & -\mathcal{A}^{T} \\ -\mathcal{A} & -B R^{-1} B^{T}\end{array}\right)$ is symmetric, $H$ is a Hamiltonian matrix and satisfies the following properties. 
Proposition 2.2.2. [13] If matrix $K$ is a Hamiltonian matrix, $p_{K}(x)$ is the characteristic polynomial of matrix $K$ and $x_{0} \in \mathbb{C}$ is a root of $p_{K}(x)=0$, then we have

$$
p_{K}\left(-x_{0}\right)=p_{K}\left(\bar{x}_{0}\right)=p_{K}\left(-\bar{x}_{0}\right)=0
$$

Theorem 2.2.3. (The real Schur-Hamiltonian decomposition) [16] Suppose

$$
M=\left(\begin{array}{cc}
A & N \\
K & -A^{T}
\end{array}\right)
$$

where $A \in \mathbb{R}^{n \times n}, N^{T}=N \in \mathbb{R}^{n \times n}$ and $K^{T}=K \in \mathbb{R}^{n \times n}$. If $M$ has no purely imaginary eigenvalues, then there exists an orthogonal matrix

$$
U=\left(\begin{array}{cc}
U_{11} & U_{12} \\
-U_{12} & U_{11}
\end{array}\right), \quad U_{11}, U_{12} \in \mathbb{R}^{n \times n}
$$

such that

$$
U^{T} M U=\left(\begin{array}{cc}
T & R \\
0 & -T^{T}
\end{array}\right), \quad T, R \in \mathbb{R}^{n \times n},
$$

where $T$ is upper quasi-triangular and $R^{T}=R$. $U$ can be chosen such that the eigenvalues of $T$ are in the open left half plane.

A block upper triangular matrix with $1 \times 1$ and $2 \times 2$ blocks on the diagonal is called upper quasi-triangular. Theorem 2.2 .3 is presented in $[16$, p. 26] and we briefly overview the proof. This result is a real valued matrix situation of Theorem 3.1 in [16, p. 19]. The authors apply an induction method in their proof. The first step is to prove the existence of unitary transformation matrix when $M$ is a $2 \times 2$ matrix. There is an error in their proof. By the condition of no purely imaginary eigenvalues, we can obtain $k \eta+\alpha^{2}>0$, not $k \eta>0$ as in $[16$, p. 20]. The second step is to assume any Hamiltonian matrices of order $2(n-1)$ without purely imaginary eigenvalues have the decomposition. The final step is to prove under the above assumption the decomposition exists for any Hamiltonian matrices of order $2 n$ without purely imaginary eigenvalues. Here the key point is to use an algorithm that is introduced in [21] to prove the eigenvalues of Hamiltonian matrix $M$ of order $2 n$ are $\lambda$ and $-\bar{\lambda}$ together with the eigenvalues of a Hamiltonian matrix $M_{1}$ of order $2(n-1)$.

Here the condition that there is no purely imaginary eigenvalues means no eigenvalues with zero real parts. Thus, zero is interpreted as a purely imaginary eigenvalue. 


\subsection{Continuous-time Algebraic Riccati Equations}

Our analysis of the SCE equation system in this thesis depends on a matrix decomposition method which involves a class of algebraic Riccati equations. In this part, we introduce a set of continuous-time algebraic Riccati equation (CARE) of the form:

$$
X D X-X \mathcal{A}-\mathcal{A}^{T} X-C=0
$$

where $\mathcal{A}, C, D$ are given matrices in $\mathbb{R}^{n \times n}$, with $C^{T}=C$ and $D^{T}=D$.

For the reader's convenience, we summarize some standard definitions on Riccati equations and linear control systems [11,23].

Definition 2.3.1. For a matrix $M \in \mathbb{R}^{n \times n}, M$ is stable if all the eigenvalues of $M$ are in the open left half-plane.

Definition 2.3.2. For a real symmetric solution $X_{+}$of the CARE (2.7), $X_{+}$is the maximal real symmetric solution if for any real symmetric solution $X,\left(X_{+}-X\right)$ is nonnegative definite.

Definition 2.3.3. For a real symmetric solution $X$ of the CARE (2.7), $X$ is an almost stabilizing solution if the eigenvalues of $(\mathcal{A}-D X)$ are in the closed left half-plane. In addition, $X$ is called a stabilizing solution if the eigenvalues of $(\mathcal{A}-D X)$ are in the open left half-plane.

Before analyzing the solution of the CARE, we first introduce some concept related to a system of the form

$$
\dot{x}=A x(t)+B u(t), \quad x(0)=x_{0} .
$$

Definition 2.3.4. For $A \in \mathbb{R}^{n \times n}$ and $B \in \mathbb{R}^{n \times m}$, a pair of matrices $(A, B)$ is called a controllable pair if

$$
\operatorname{rank}\left[\begin{array}{lllll}
B & A B & A^{2} B & \ldots & A^{n-1} B
\end{array}\right]=n
$$

We say the system (2.8) is controllable if, for any fixed $x_{0}$ and $x_{1}$, there exists a continuous control $u(t)$ such that $x(t)$ satisfies $(2.8)$ and the terminal state $x(T)=x_{1}$, for terminal time $T>0$. In addition, it can be proved that the system (2.8) is controllable is equivalent to that the pair $(A, B)$ is controllable $[11]$. 
Definition 2.3.5. For $A \in \mathbb{R}^{n \times n}$ and $B \in \mathbb{R}^{n \times m}$, a pair of matrices $(A, B)$ is called a stabilizable pair if there exists a matrix $K \in \mathbb{R}^{m \times n}$ such that $A+B K$ is stable (i.e. all eigenvalues of $A+B K$ are in the open left half-plane).

The system (2.8) is stabilizable means for any fixed initial state $x_{0}$, there exists a continuous control $u(t)$ such that $x(t)$ satisfies $(2.8)$ and $\lim _{t \rightarrow+\infty} x(t)=0$. In parallel to controllability, the system is stabilizable if and only if the pair $(A, B)$ is stabilizable.

Theorem 2.3.6. [11, p. 90] If the pair $(A, B)$ is controllable, then the pair $(A, B)$ is stabilizable.

Example 2.3.7. Let $x \in \mathbb{R}, \dot{x}=-1.1 x+0 u$.

This system is not controllable but stabilizable. Indeed, since for fixed initial state $x_{0}$, the solution $x(t)$ is always a fixed trajectory no matter what the control $u(t)$ is. Thus, for a given $T, x(T)$ is fixed, which means uncontrollability. However, since $A=-1.1$ is a stable matrix, the solution $x(t)$ is stable (i.e. $\lim _{t \rightarrow+\infty} x(t)=0$ ), which means this system is stabilizable.

Theorem 2.3.6 shows that controllable systems must be stabilizable. Furthmore, Example 2.3.7 shows that stabilizable systems may be uncontrollable. Thus, controllability is a stronger condition. Actually, all the following theorems are proved to be correct under the controllability condition firstly, and then we can relax the condition to the stabilizability condition by selecting a special orthogonal matrix to transform. The details are as follow.

Considering a space $\mathcal{C}:=\operatorname{Im}\left[\begin{array}{lllll}D & \mathcal{A} D & \mathcal{A}^{2} D & \ldots & \mathcal{A}^{n-1} D\end{array}\right]$, where Im means the subspace spanned by the columns. By $[11$, p. 85], $\mathcal{C}$ is $\mathcal{A}$-invariant. By the discussion in $[11, \mathrm{p}$. 193], there exists a set of orthogonal basis $T=\left(\begin{array}{ll}T_{1} & T_{2}\end{array}\right)$, where $T_{1} \in \mathcal{C}$ and $T_{2} \in \mathcal{C}^{\perp}$, such that

$$
T^{T} A T=\left(\begin{array}{cc}
\mathcal{A}_{1} & \mathcal{A}_{12} \\
0 & \mathcal{A}_{2}
\end{array}\right), T^{T} D T=\left(\begin{array}{cc}
D_{1} & 0 \\
0 & 0
\end{array}\right), T^{T} C T=\left(\begin{array}{cc}
C_{1} & C_{12} \\
C_{12}^{T} & C_{2}
\end{array}\right)
$$

where $D_{1} \geq 0 ; C_{1}, C_{2}$ is symmetric; $\left(\mathcal{A}_{1}, D_{1}\right)$ is controllable and $\mathcal{A}_{2}$ is stable. If we set $Y=T^{T} X T$ and $X$ is real symmetric, (2.7) is equivalent to

$$
Y\left(T^{T} D T\right) Y-Y\left(T^{T} \mathcal{A} T\right)-\left(T^{T} \mathcal{A} T\right)^{T} Y-T^{T} C T=0 .
$$


Thus, if our interest is the real symmetric solution of (2.7), we can assume:

$$
\mathcal{A}=\left(\begin{array}{cc}
\mathcal{A}_{1} & \mathcal{A}_{12} \\
0 & \mathcal{A}_{2}
\end{array}\right), \quad D=\left(\begin{array}{cc}
D_{1} & 0 \\
0 & 0
\end{array}\right), \quad C=\left(\begin{array}{cc}
C_{1} & C_{12} \\
C_{12}^{T} & C_{2}
\end{array}\right)
$$

Thus, letting $X$ be a block matrix

$$
\left(\begin{array}{cc}
X_{1} & X_{12} \\
X_{12}^{T} & X_{2}
\end{array}\right)
$$

where $X_{1}$ and $\mathcal{A}_{1}$ have the same dimension, (2.7) is equivalent to the following equations:

$$
\begin{gathered}
X_{1} D_{1} X_{1}-X_{1} \mathcal{A}_{1}-\mathcal{A}_{1}^{T} X_{1}-C_{1}=0 \\
\left(\mathcal{A}_{1}^{T}-X_{1} D_{1}\right) X_{12}+X_{12} \mathcal{A}_{2}+\left(C_{12}+X_{1} \mathcal{A}_{12}\right)=0 \\
-\mathcal{A}_{2}^{T} X_{2}-X_{2} \mathcal{A}_{2}+X_{12}^{T} D_{1} X_{12}-X_{12}^{T} \mathcal{A}_{12}-\mathcal{A}_{12}^{T} X_{12}-C_{12}=0 .
\end{gathered}
$$

Theorem 2.3.8. [11, p. 195] If $D \geq 0,(\mathcal{A}, D)$ is stabilizable and there is a real symmetric solution of (2.7), then there is a maximal real symmetric solution $X_{+}$.

Theorem 2.3.9. $[11, p$. 196] Suppose that $D \geq 0,(\mathcal{A}, D)$ is stabilizable and there is a real symmetric solution of (2.7). Then for the maximal real symmetric solution $X_{+}$of (2.7), $\mathcal{A}-D X_{+}$is stable if and only if the matrix $M:=\left(\begin{array}{cc}\mathcal{A} & -D \\ -C & -\mathcal{A}^{T}\end{array}\right)$ has no eigenvalues on the imaginary axis.

In $[11$, p. 196], there is a proof when the coefficient matrices $\mathcal{A}, C, D$ are complex. Here, for the reader's convenience, we provide a proof of Theorem 2.3.9 when the coefficient matrices are real.

Proof. Without loss of generality, we can assume the coefficient matrices $\mathcal{A}, C, D$ have the form of (2.9). 
1) The "if" part. Suppose $M$ has no eigenvalues on the imaginary axis, and $M$ has the form:

$$
\left(\begin{array}{cccc}
\mathcal{A}_{1} & \mathcal{A}_{12} & -D_{1} & 0 \\
0 & \mathcal{A}_{2} & 0 & 0 \\
-C_{1} & -C_{12} & -\mathcal{A}_{1}^{T} & 0 \\
-C_{12}^{T} & -C_{2} & -\mathcal{A}_{12} & -\mathcal{A}_{2}^{T}
\end{array}\right)
$$

By orthogonal transformation matrix $T=\left(\begin{array}{cccc}I & 0 & 0 & 0 \\ 0 & 0 & I & 0 \\ 0 & I & 0 & 0 \\ 0 & 0 & 0 & I\end{array}\right)$, we have

$$
T^{T} M T=\left(\begin{array}{cccc}
\mathcal{A}_{1} & -D_{1} & \mathcal{A}_{12} & 0 \\
-C_{1} & -\mathcal{A}_{1}^{T} & -C_{12} & 0 \\
0 & 0 & \mathcal{A}_{2} & 0 \\
-C_{12}^{T} & -\mathcal{A}_{12} & -C_{2} & -\mathcal{A}_{2}^{T}
\end{array}\right)
$$

From (2.14), we can obtain the eigenvalues of $M$ consisting of the eigenvalues of $\mathcal{A}_{2}$, $-\mathcal{A}_{2}^{T}$ and $M_{1}$, where

$$
M_{1}=\left(\begin{array}{cc}
\mathcal{A}_{1} & -D_{1} \\
-C_{1} & -\mathcal{A}_{1}^{T}
\end{array}\right)
$$

Thus, $M_{1}$ has no eigenvalues on the imaginary axis. According to [11, Theorem 7.5.1, p. 168], since $\left(\mathcal{A}_{1}, D_{1}\right)$ is controllable and $M_{1}$ has no eigenvalues on the imaginary, there exists a solution $X_{1+}$ of $(2.11)$ such that $\mathcal{A}-D_{1} X_{1+}$ is stable. Let $X_{+}=\left(\begin{array}{cc}X_{1+} & X_{12+} \\ X_{12+}^{T} & X_{2+}\end{array}\right)$, where $X_{12+}$ and $X_{2+}$ are the corresponding solution of (2.12) and (2.13).

From (2.9) and (2.10), we have

$$
\mathcal{A}-D X_{+}=\left(\begin{array}{cc}
\mathcal{A}_{1}-D_{1} X_{1+} & \mathcal{A}_{12}-D_{1} X_{12+} \\
0 & \mathcal{A}_{2}
\end{array}\right)
$$

The eigenvalues of $\mathcal{A}-D X_{+}$consist of the eigenvalue of $\mathcal{A}_{1}-D_{1} X_{1+}$ and $\mathcal{A}_{2}$, which are both stable. Thus, $\mathcal{A}-D X_{+}$is stable. 
2) The "only if" part. Suppose $\mathcal{A}-D X_{+}$are stable. Since $\mathcal{A}_{2}$ is stable, then the eigenvalues of $-\mathcal{A}_{2}^{T}$ are all in the open right half-plane. We just need to prove $M_{1}$ has no eigenvalues on the imaginary axis. Let $X_{1+}$ be the corresponding block matrix of $X_{+}$, and it is obvious that $X_{1+}$ is the maximal solution of (2.11). From (2.15), since $\mathcal{A}-D X_{+}$is stable and $\mathcal{A}_{2}$ is stable, $\mathcal{A}_{1}-D_{1} X_{1+}$ must be stable.

Consider an invertible transformation

$$
T=\left(\begin{array}{cc}
I & 0 \\
X_{1+} & I
\end{array}\right), \quad T^{-1}=\left(\begin{array}{cc}
I & 0 \\
-X_{1+} & I
\end{array}\right)
$$

Then $T^{-1} M_{1} T=\left(\begin{array}{cc}\mathcal{A}_{1}-D_{1} X_{1+} & -D_{1} \\ 0 & -\left(\mathcal{A}_{1}-D_{1} X_{1+}\right)^{T}\end{array}\right)$. Since $\mathcal{A}_{1}-D_{1} X_{1+}$ is stable, then the eigenvalues of $-\left(\mathcal{A}_{1}-D_{1} X_{1+}\right)^{T}$ are all in the open right half-plane, so $T^{-1} M_{1} T$ has no eigenvalues on the imaginary axis. In addition, $M_{1}$ shares the same eigenvalues with $T^{-1} M_{1} T$. Thus $M_{1}$ has no eigenvalues on the imaginary axis.

Definition 2.3.10. For a matrix $M \in \mathbb{R}^{n \times n}$ and its eigenvalue $\lambda_{0}$, the sizes of its Jordan canonical block in the Jordan canonical form of $M$ is called partial multiplicities of $\lambda_{0}$.

Example 2.3.11. Consider

$$
M=\left(\begin{array}{cccccccccc}
1 & 1 & 0 & 0 & 0 & 0 & 0 & 0 & 0 & 0 \\
0 & 1 & 1 & 0 & 0 & 0 & 0 & 0 & 0 & 0 \\
0 & 0 & 1 & 0 & 0 & 0 & 0 & 0 & 0 & 0 \\
0 & 0 & 0 & 1 & 0 & 0 & 0 & 0 & 0 & 0 \\
0 & 0 & 0 & 0 & 1 & 0 & 0 & 0 & 0 & 0 \\
0 & 0 & 0 & 0 & 0 & -1 & 1 & 0 & 0 & 0 \\
0 & 0 & 0 & 0 & 0 & 0 & -1 & 1 & 0 & 0 \\
0 & 0 & 0 & 0 & 0 & 0 & 0 & -1 & 1 & 0 \\
0 & 0 & 0 & 0 & 0 & 0 & 0 & 0 & -1 & 1 \\
0 & 0 & 0 & 0 & 0 & 0 & 0 & 0 & 0 & -1
\end{array}\right) .
$$

This matrix $M$ has two eigenvalues 1 and -1 . For the eigenvalue 1 , its partial multiplicities are 3, 1 and 1 . For the eigenvalue -1 , its partial multiplicities are 5 . 
Theorem 2.3.12. [11, p. 239] There exists a unique almost stabilizing solution of the CARE (2.7) if and only if $(\mathcal{A}, D)$ is stabilizable and the partial multiplicities of purely imaginary (or zero) eigenvalues of the matrix $M:=\left(\begin{array}{cc}\mathcal{A} & -D \\ -C & -\mathcal{A}^{T}\end{array}\right)$ are all even.

\subsection{Frequency Domain Condition}

In this part, we consider a special form of the CARE:

$$
X B R^{-1} B^{T} X-\mathcal{A}^{T} X-X \mathcal{A}-C=0
$$

where $\mathcal{A}, Q \in \mathbb{R}^{n \times n}, B \in \mathbb{R}^{n \times m}, R \in \mathbb{R}^{m \times m} ; C$ is symmetric and $R$ is positive definite.

Firstly, we introduce the Frequency-Domain Inequality (FDI) [22]:

$$
\hat{H}(\bar{s}, s)=R+B^{T}\left(I \bar{s}-\mathcal{A}^{T}\right)^{-1} C(I s-\mathcal{A})^{-1} B \geq 0 .
$$

The following theorem is related to FDI.

Theorem 2.4.1. [22] Assume that the pair $(\mathcal{A}, B)$ is controllable. Then the following are true.

1) Existence: CARE (2.16) has a real symmetric solution if and only if $\hat{H}(-j \omega, j \omega) \geq 0$ for all real $w$. There always exists exactly one such solution, denoted by $X_{+}$, which has the additional property that $\operatorname{Re} \lambda\left(\mathcal{A}-B R^{-1} B^{T} X_{+}\right) \leq 0$, i.e. $X_{+}$is the almost stabilizing solution of CARE (2.16).

2) Strict Inequality: the strict inequality

$$
\hat{H}(-j \omega, j \omega) \geq \epsilon B^{T}\left(-I j \omega-\mathcal{A}^{T}\right)^{-1}(I j \omega-\mathcal{A})^{-1} B
$$

holds, for some $\epsilon>0$ if and only if the CARE (2.16) has a real symmetric solution $X_{+}$, such that $\mathcal{R} e \lambda\left(\mathcal{A}-B R^{-1} B^{T} X_{+}\right)<0$, i.e. $X_{+}$is the stabilizing solution of CARE (2.16).

Example 2.4.2. Consider

$$
H=\left(\begin{array}{cc}
a-b^{2} \Pi / r-\rho / 2 & -b^{2} / r \\
\left(2 \gamma-\gamma^{2}\right) q & -\left(a-b^{2} \Pi / r-\rho / 2\right)
\end{array}\right)
$$


where

$$
\rho \Pi=2 a \Pi-b^{2} \Pi / r+q .
$$

Actually this matrix is the size $2 \times 2$ matrix of (2.4), where $\mathcal{A}=a-b^{2} \Pi / r-\rho / 2, B=b$, $R=r \geq 0$ and $Q_{\Gamma}=\left(2 \gamma-\gamma^{2}\right) q$, where $q>0$ and $0 \leq \gamma \leq 1$.

From (2.19), denote $(a-\rho / 2)$ as $a_{\rho}$ and $b^{2} / r$ as $b_{r}$; then we can obtain that $\Pi=$ $a_{\rho} / b_{r} \pm \sqrt{a_{\rho}^{2} / b_{r}^{2}+q / b_{r}}$ and choose the greater one.

Firstly, consider the following CARE:

$$
X B R^{-1} B^{T} X-\mathcal{A}^{T} X-X \mathcal{A}+Q_{\Gamma}=0
$$

and then the corresponding FDI is $\hat{H}(-j \omega, j \omega)=r-q b^{2}\left(2 \gamma-\gamma^{2}\right) /\left(w^{2}+a_{\rho}^{2}+q b / r^{2}\right) \geq 0$. Then we can obtain the strict FDI:

$$
1-q b_{r}^{2}\left(2 \gamma-\gamma^{2}\right) /\left(w^{2}+a_{\rho}^{2}+q b_{r}\right) \geq \epsilon b_{r}^{2} /\left(w^{2}+a_{\rho}^{2}+q b_{r}\right)
$$

which is equivalent to

$$
\omega^{2}+a_{\rho}^{2}+q b_{r}(\gamma-1)^{2} \geq \epsilon b_{r}
$$

If for all $\omega \in R$ and some $\epsilon>0,(2.20)$ holds, it means $a_{\rho} \neq 0$ or $\left\{\gamma \neq 1\right.$ and $\left.b_{r} \neq 0\right\}$.

On the other hand, letting

$$
\operatorname{det}(\lambda I-H)=\operatorname{det}\left(\begin{array}{cc}
\lambda+\sqrt{a_{\rho}^{2}+q b_{r}} & b_{r} \\
\left(\gamma^{2}-2 \gamma\right) q & \lambda-\sqrt{a_{\rho}^{2}+q b_{r}}
\end{array}\right)=0,
$$

we can obtain $\lambda^{2}=a_{\rho}^{2}+q b_{r}(\gamma-1)^{2}$. If the matrix $H$ has no purely imaginary eigenvalues, it is equivalent to $a_{\rho}^{2}+q b_{r}(\gamma-1)^{2}>0$, i.e. $a_{\rho} \neq 0$ or $\left\{\gamma \neq 1\right.$ and $\left.b_{r} \neq 0\right\}$.

Here, we find that $H$ has no purely imaginary eigenvalues is equivalent to that the corresponding strict FDI holds when $H$ is a $2 \times 2$ matrix. 


\subsection{Existence and Uniqueness of A Solution}

In Section 2.1, we transform the SCE equation system (2.1)-(2.2) to the following Hamiltonian system:

$$
\left(\begin{array}{c}
\frac{d \tilde{x}}{d t} \\
\frac{d \tilde{s}}{d t}
\end{array}\right)=H\left(\begin{array}{c}
\tilde{x} \\
\tilde{s}
\end{array}\right)+\left(\begin{array}{c}
0 \\
\tilde{\eta}_{\Gamma}
\end{array}\right)
$$

where $\mathcal{A}=A-B R^{-1} B^{T} \Pi-\frac{\rho}{2} I$ and

$$
H=\left(\begin{array}{cc}
\mathcal{A} & -B R^{-1} B^{T} \\
Q_{\Gamma} & -\mathcal{A}^{T}
\end{array}\right)
$$

Now we develop a new method to analyze the solution of the SCE equation system (2.1)(2.2) by using the properties of Hamiltonian matrices and the continuous-time algebraic Riccati equation.

Lemma 2.5.1. If there exists an invertible matrix $U=\left(\begin{array}{cc}U_{11} & U_{12} \\ U_{21} & U_{22}\end{array}\right)$, where $U_{11}$ is invertible and

$$
U^{-1} H U=\left(\begin{array}{cc}
\mathcal{A}_{C} & F \\
0 & -\mathcal{A}_{C}^{T}
\end{array}\right),
$$

where $\mathcal{A}_{C}$ is stable, we can obtain the following assertions:

1) For any fixed initial state $x_{0}$ of SCE equation system (2.1)-(2.2), there exists an initial condition $s_{0}$, such that SCE equation system (2.1)-(2.2) has a unique solution $(\bar{x}, s) \in$ $C_{\rho / 2}\left([0, \infty), \mathbb{R}^{2 n}\right)$.

2) Such an initial condition $s_{0}$ is unique and is equal to

$$
U_{21} U_{11}^{-1} \bar{x}_{0}+\left(U_{21} U_{11}^{-1} U_{12}-U_{22}\right) \int_{0}^{+\infty} e^{A_{C}^{T} \tau} V_{22} \eta_{\Gamma} e^{-\rho \tau / 2} d \tau
$$

where $V=U^{-1}=\left(\begin{array}{ll}V_{11} & V_{12} \\ V_{21} & V_{22}\end{array}\right)$.

Proof. 1) Proof of existence. 
Let $\left(\begin{array}{l}\hat{x} \\ \hat{s}\end{array}\right)=V\left(\begin{array}{l}\tilde{x} \\ \tilde{s}\end{array}\right)$. The ODE systems of $(\hat{x}, \hat{s})$ is:

$$
\begin{aligned}
& \frac{d \hat{x}}{d t}=\mathcal{A}_{C} \hat{x}+F \hat{s}+V_{12} \tilde{\eta}_{\Gamma}, \\
& \frac{d \hat{s}}{d t}=-\mathcal{A}_{C}^{T} \hat{s}+V_{22} \tilde{\eta}_{\Gamma} .
\end{aligned}
$$

Firstly, since $\tilde{\eta}_{\Gamma}=e^{-\rho t / 2} \eta_{\Gamma}$, from (2.23), we can obtain that

$$
\frac{d \hat{s}}{d t}=-\mathcal{A}_{C}^{T} \hat{s}+V_{22} \eta_{\Gamma} e^{-\rho t / 2}
$$

Thus, the solution of ODE (2.23) has the form:

$$
\hat{s}(t)=e^{-\mathcal{A}_{C}^{T} t} \hat{s}_{0}+\int_{0}^{t} e^{-\mathcal{A}_{C}^{T}(t-\tau)} V_{22} \eta_{\Gamma} e^{-\rho \tau / 2} d \tau
$$

Since $\mathcal{A}_{C}^{T}$ is stable, the integral $\int_{0}^{+\infty} e^{\mathcal{A}_{C}^{T} \tau} V_{22} \eta_{\Gamma} e^{-\rho \tau / 2} d \tau$ is finite. Let

$$
\hat{s}_{0}=-\int_{0}^{+\infty} e^{\mathcal{A}_{C}^{T} \tau} V_{22} \eta_{\Gamma} e^{-\rho \tau / 2} d \tau
$$

Then we obtain that

$$
\begin{aligned}
\hat{s}(t) & =-\int_{t}^{\infty} e^{-\mathcal{A}_{C}^{T}(t-\tau)} V_{22} \eta_{\Gamma} e^{-\rho \tau / 2} d \tau \\
& =-\int_{0}^{\infty} e^{\mathcal{A}_{C}^{T} \tau} V_{22} \eta_{\Gamma} e^{-\rho(\tau+t) / 2} d \tau \\
& =-e^{-\rho t / 2} \int_{0}^{\infty} e^{\left(\mathcal{A}_{C}^{T}-\frac{\rho}{2} I\right) \tau} V_{22} \eta_{\Gamma} d \tau .
\end{aligned}
$$

Denote the maximal real part of eigenvalues of $\mathcal{A}_{C}$ as $\sigma_{s}$. Since $\mathcal{A}_{C}$ is stable, $\sigma_{s}$ is negative. Consider a real positive number $\varepsilon$ such that $\varepsilon<\min \left(-\sigma_{s}, \frac{\rho}{2}\right)$. From equation (2.25), we can obtain

$$
e^{\varepsilon t} \hat{s}(t)=-e^{-\left(\frac{\rho}{2}-\varepsilon\right) t} \int_{0}^{\infty} e^{\left(\mathcal{A}_{C}^{T}-\frac{\rho}{2} I\right) \tau} V_{22} \eta_{\Gamma} d \tau
$$

Since $\mathcal{A}_{C}^{T}-\frac{\rho}{2} I$ is stable and $\frac{\rho}{2}-\varepsilon>0$, we have $e^{\varepsilon t} \hat{s}(t)$ is bounded. Thus, $e^{\frac{\rho}{2} t} \hat{s}(t)$ is within $C_{\rho / 2}\left([0, \infty), \mathbb{R}^{n}\right)$.

Secondly, from the ODE (2.22), we can obtain that

$$
\frac{d \hat{x}}{d t}=\mathcal{A}_{C} \hat{x}+F \hat{s}(t)+V_{12} \eta_{\Gamma} e^{-\rho t / 2},
$$


where $\hat{s}(t)$ is the bounded solution $(2.25)$.

Now consider $e^{\varepsilon t} \hat{x}(t)$, where $\hat{x}(t)$ is the solution of ODE (2.27). We can obtain that

$$
\begin{aligned}
\frac{d\left[e^{\varepsilon t} \hat{x}(t)\right]}{d t} & =\varepsilon e^{\varepsilon t} \hat{x}(t)+e^{\varepsilon t} \frac{d \hat{x}}{d t} \\
& =\varepsilon e^{\varepsilon t} \hat{x}(t)+e^{\varepsilon t}\left[\mathcal{A}_{C} \hat{x}(t)+F \hat{s}(t)+V_{12} \eta_{\Gamma} e^{-\rho t / 2}\right] \\
& =\left(\mathcal{A}_{C}+\varepsilon I\right)\left[e^{\varepsilon t} \hat{x}(t)\right]+F\left[e^{\varepsilon t} \hat{s}(t)\right]+V_{12} \eta_{\Gamma} e^{\left(\varepsilon-\frac{\rho}{2}\right) t}
\end{aligned}
$$

From (2.26), we can obtain that $\lim _{t \rightarrow+\infty} e^{\varepsilon t} \hat{s}(t)=0$. In addition, since the $\left(\mathcal{A}_{C}+\varepsilon I\right)$ is stable, for any initial condition $\hat{x}_{0}$, we conclude that $e^{\varepsilon t} \hat{x}(t)$ is bounded, which means that $e^{\frac{\rho}{2} t} \hat{x}(t)$ is within $C_{\rho / 2}\left([0, \infty), \mathbb{R}^{n}\right)$.

Now, we obtain $e^{\frac{\rho}{2} t} \hat{x}(t)$ and $e^{\frac{\rho}{2} t} \hat{s}(t)$ are both within $C_{\rho / 2}\left([0, \infty), \mathbb{R}^{n}\right)$. Since

$$
\left(\begin{array}{l}
\bar{x}(t) \\
s(t)
\end{array}\right)=U\left(\begin{array}{c}
e^{\frac{\rho}{2} t} \hat{x}(t) \\
e^{\frac{\rho}{2} t} \hat{s}(t)
\end{array}\right),
$$

we obtain that $\bar{x}(t) \in C_{\rho / 2}\left([0, \infty), \mathbb{R}^{n}\right)$ and $s(t) \in C_{\rho / 2}\left([0, \infty), \mathbb{R}^{n}\right)$, i.e. $(\bar{x}, s) \in C_{\rho / 2}\left([0, \infty), \mathbb{R}^{2 n}\right)$.

Finally, since

$$
\begin{aligned}
& \bar{x}_{0}=U_{11} \hat{x}_{0}+U_{12} \hat{s}_{0}, \\
& s_{0}=U_{21} \hat{x}_{0}+U_{22} \hat{s}_{0},
\end{aligned}
$$

and $U_{11}$ is invertible, for any fixed $\bar{x}_{0}$ and fixed $\hat{s}_{0}$, there always exists an initial condition

$$
s_{0}=U_{21} U_{11}^{-1}\left(\bar{x}_{0}-U_{12} \hat{s}_{0}\right)+U_{22} \hat{s}_{0} .
$$

Thus, for obtaining $\bar{x} \in C_{\rho / 2}\left([0, \infty), \mathbb{R}^{n}\right)$ and $s \in C_{\rho / 2}\left([0, \infty), \mathbb{R}^{n}\right)$, we can choose the initial condition

$$
\begin{aligned}
s_{0} & =U_{21} U_{11}^{-1}\left(\bar{x}_{0}-U_{12} \hat{s}_{0}\right)+U_{22} \hat{s}_{0} \\
& =U_{21} U_{11}^{-1} \bar{x}_{0}-\left(U_{21} U_{11}^{-1} U_{12}-U_{22}\right) \hat{s}_{0} \\
& =U_{21} U_{11}^{-1} \bar{x}_{0}+\left(U_{21} U_{11}^{-1} U_{12}-U_{22}\right) \int_{0}^{+\infty} e^{\mathcal{A}_{C}^{T} \tau} V_{22} \eta_{\Gamma} e^{-\rho \tau / 2} d \tau
\end{aligned}
$$

2) Proof of uniqueness. 
Assume there exists another initial condition $s_{0}^{\prime}$ such that the corresponding solution $\left(\bar{x}^{\prime}(t), s^{\prime}(t)\right) \in C_{\rho / 2}\left([0, \infty), \mathbb{R}^{2 n}\right)$. Since

$$
\begin{aligned}
& \bar{x}_{0}=U_{11} \hat{x}_{0}^{\prime}+U_{12} \hat{s}_{0}^{\prime}, \\
& s_{0}^{\prime}=U_{21} \hat{x}_{0}^{\prime}+U_{22} \hat{s}_{0}^{\prime},
\end{aligned}
$$

and $U$ is invertible, we can obtain the corresponding $\hat{s}_{0}^{\prime}$.

Now we denote the corresponding solution of $\operatorname{ODE}(2.24)$ as $\hat{s}^{\prime}(t)$, where the initial condition of ODE $(2.24)$ is $\hat{s}_{0}^{\prime}$. Thus, $\hat{s}^{\prime}(t)$ has the following form:

$$
\hat{s}^{\prime}(t)=e^{-\mathcal{A}_{C}^{T} t} \hat{s}_{0}^{\prime}+\int_{0}^{t} e^{-\mathcal{A}_{C}^{T}(t-\tau)} V_{22} \eta_{\Gamma} e^{-\rho \tau / 2} d \tau
$$

Since $s^{\prime}(t) \in C_{\rho / 2}\left([0, \infty), \mathbb{R}^{n}\right)$, from the first part of proof, we can obtain that $\hat{s}^{\prime}(t)$ must be bounded. In addition, since $\hat{s}(t)$ is also bounded, we can obtain $\hat{s}(t)-\hat{s}^{\prime}(t)$ is bounded.

From (2.25) and (2.33), we have

$$
\hat{s}(t)-\hat{s}^{\prime}(t)=e^{-\mathcal{A}_{C}^{T} t}\left(\hat{s}_{0}-\hat{s}_{0}^{\prime}\right) .
$$

Since the eigenvalues of $-\mathcal{A}_{C}^{T}$ are all in the open right half-plane, $\hat{s}_{0}-\hat{s}_{0}^{\prime}$ must be 0 and otherwise the RHS of (2.34) is not bounded. Thus, $\hat{s}_{0}^{\prime}=\hat{s}_{0}$.

According to equations (2.31)-(2.32) and the invertibility of $U_{11}$, we can obtain that that

$$
\begin{aligned}
s_{0}^{\prime} & =U_{21} U_{11}^{-1}\left(\bar{x}_{0}-U_{12} \hat{s}_{0}^{\prime}\right)+U_{22} \hat{s}_{0}^{\prime} \\
& =U_{21} U_{11}^{-1}\left(\bar{x}_{0}-U_{12} \hat{s}_{0}\right)+U_{22} \hat{s}_{0} \\
& =s_{0} .
\end{aligned}
$$

Thus, the initial condition $s_{0}$ of SCE equation system (2.1)-(2.2) such that $(\bar{x}(t), s(t)) \in$ $C_{\rho / 2}\left([0, \infty), \mathbb{R}^{2 n}\right)$ is unique. It is obvious that the solution $(\bar{x}, s)$ within $C_{\rho / 2}\left([0, \infty), \mathbb{R}^{2 n}\right)$ is unique.

Theorem 2.5.2. Assume the pair $(A, B)$ is controllable and this inequaity

$$
R+B^{T}\left(-I j \omega-\mathcal{A}^{T}\right)^{-1}\left(-Q_{\Gamma}\right)(I j \omega-\mathcal{A})^{-1} B \geq \epsilon B^{T}\left(-I j \omega-\mathcal{A}^{T}\right)^{-1}(I j \omega-\mathcal{A})^{-1} B
$$


holds, for some $\epsilon>0$. Then there exists a unique initial condition $s_{0}$, such that the $S C E$ equation system (2.1)-(2.2) has a unique solution $(\bar{x}, s) \in C_{\rho / 2}\left([0, \infty), \mathbb{R}^{2 n}\right)$.

Proof. Consider a CARE:

$$
X B R^{-1} B^{T} X-X \mathcal{A}-\mathcal{A}^{T} X+Q_{\Gamma}=0 .
$$

Since $(A, B)$ is controllable, $(\mathcal{A}, B)$ is controllable [23]. According to Theorem 2.4.1, there exists a solution $X_{+}$of CARE (2.36) such that $\left(\mathcal{A}-B R^{-1} B^{T} X_{+}\right)$is stable.

$$
\begin{aligned}
& \text { Setting } U=\left(\begin{array}{rr}
I & 0 \\
X_{+} & I
\end{array}\right), U \text { is an invertible matrix and } U^{-1}=\left(\begin{array}{cc}
I & 0 \\
-X_{+} & I
\end{array}\right) . \\
&\left.\begin{array}{rl}
U^{-1} H U & =\left(\begin{array}{cr}
I & 0 \\
-X_{+} & I
\end{array}\right)\left(\begin{array}{cc}
\mathcal{A} & -B R^{-1} B^{T} \\
Q_{\Gamma} & -\mathcal{A}^{T}
\end{array}\right)\left(\begin{array}{cc}
I & 0 \\
X_{+} & I
\end{array}\right) \\
& =\left(\begin{array}{c}
\mathcal{A}-B R^{-1} B^{T} X_{+} \\
X_{+} B R^{-1} B^{T} X_{+}-X_{+} A-A^{T} X_{+}+Q_{\Gamma}
\end{array}-\left(\mathcal{A}-B R^{-1} B^{T} X_{+}\right)^{T}\right.
\end{array}\right) \\
&=\left(\begin{array}{cc}
\mathcal{A}_{C} & -B R^{-1} B^{T} \\
0 & -\mathcal{A}_{C}^{T}
\end{array}\right),
\end{aligned}
$$

where $\mathcal{A}_{C}=\mathcal{A}-B R^{-1} B^{T} X_{+}$is stable.

According to Lemma 2.5.1, by selecting the initial condition

$$
s_{0}=X_{+} \bar{x}_{0}-\int_{0}^{+\infty} e^{\mathcal{A}_{C}^{T} \tau} \eta_{\Gamma} e^{-\rho \tau / 2} d \tau
$$

there exists a solution $(\bar{x}, s) \in C_{\rho / 2}\left([0, \infty), \mathbb{R}^{2 n}\right)$. In addition, such an initial condition and such a solution are both unique.

Theorem 2.5.2 guarantees that for a controllable system, if the corresponding coefficient matrices satisfy the strict frequency domain inequality, we can choose an explicit initial condition $s_{0}$, such that the corresponding solution $(\bar{x}, s) \in C_{\rho / 2}\left([0, \infty), \mathbb{R}^{2 n}\right)$. Notice that $Q_{\Gamma}$ in Theorem 2.5.2 corresponds to $-C$ in Theorem 2.4.1.

Recall Example 2.4.2:

$$
H=\left(\begin{array}{cc}
a-b^{2} \Pi / r-\rho / 2 & -b^{2} / r \\
\left(2 \gamma-\gamma^{2}\right) q & -\left(a-b^{2} \Pi / r-\rho / 2\right)
\end{array}\right)
$$


For matrix $H$, it has no purely imaginary eigenvalues if and only if the corresponding strict FDI holds. Since matrix $H$ in Example 2.4.2 is the $2 \times 2$ dimension matrix $H$ in Theorem 2.5.1, it is reasonable for us to try to replace the frequency domain inequality condition (2.35) by an eigenvalue condition on $H$, as an alternative requirement on the model.

On the other hand, for $b \neq 0$, the pair $(a, b)$ is always controllable. However, for the matrix pair $(A, B)$, controllability is restrictive. Thus, it is desirable to relax the condition on the pair $(A, B)$.

Theorem 2.5.3. Assume that the pair $(A, B)$ is stabilizable and matrix $H$ has no purely imaginary eigenvalues, then there exists a unique initial condition $s_{0}$, such that the SCE equation system (2.1)-(2.2) has a unique solution $(\bar{x}, s) \in C_{\rho / 2}\left([0, \infty), \mathbb{R}^{2 n}\right)$.

Proof. Since $\mathcal{A}$ is stable, $\left(\mathcal{A}, B R^{-1} B^{T}\right)$ is stabilizable.

For matrix $H=\left(\begin{array}{cc}\mathcal{A} & -B R^{-1} B^{T} \\ Q_{\Gamma} & -\mathcal{A}^{T}\end{array}\right)$, it has no purely imaginary eigenvalues, which means the partial multiplicities of pure imaginary eigenvalue are all 0 . Consider the following CARE

$$
X B R^{-1} B^{T} X-X \mathcal{A}-\mathcal{A}^{T} X+Q_{\Gamma}=0
$$

According to Theorem 2.3.12, there exists a unique almost stabilizing solution of CARE (2.40). Furthermore, CARE (2.40) satisfies the condition of Theorem 2.3.9. Thus, according to Theorem 2.3.9, there exists a maximal real symmetric solution $X_{+}$, such that $\mathcal{A}-$ $B R^{-1} B^{T} X_{+}$is stable. Actually since $X_{+}$is also the almost stabilizing solution, by Theorem 2.3.12, $X_{+}$is unique.

$$
\text { Setting } \begin{aligned}
U & =\left(\begin{array}{rr}
I & 0 \\
X_{+} & I
\end{array}\right), U \text { is an invertible matrix and } U^{-1}=\left(\begin{array}{cc}
I & 0 \\
-X_{+} & I
\end{array}\right) \text {. Then } \\
U^{-1} H U & =\left(\begin{array}{cr}
I & 0 \\
-X_{+} & I
\end{array}\right)\left(\begin{array}{cc}
\mathcal{A} & -B R^{-1} B^{T} \\
Q_{\Gamma} & -\mathcal{A}^{T}
\end{array}\right)\left(\begin{array}{cc}
I & 0 \\
X_{+} & I
\end{array}\right) \\
& =\left(\begin{array}{cc}
\mathcal{A}-B R^{-1} B^{T} X_{+} \\
X_{+} B R^{-1} B^{T} X_{+}-X_{+} A-A^{T} X_{+}+Q_{\Gamma} & -\left(\mathcal{A}-B R^{-1} B^{T} X_{+}\right)^{T}
\end{array}\right) \\
& =\left(\begin{array}{cc}
\mathcal{A}_{C} & -B R^{-1} B^{T} \\
0 & -\mathcal{A}_{C}^{T}
\end{array}\right),
\end{aligned}
$$


where $\mathcal{A}_{C}=\mathcal{A}-B R^{-1} B^{T} X_{+}$is stable. According to Lemma 2.5.1, by selecting the initial

condition $s_{0}=X_{+} \bar{x}_{0}-\int_{0}^{+\infty} e^{\mathcal{A}_{C}^{T} \tau} \eta_{\Gamma} e^{-\rho \tau / 2} d \tau$, there exists a solution $(\bar{x}, s) \in C_{\rho / 2}\left([0, \infty), \mathbb{R}^{2 n}\right)$. In addition, such an initial condition and such a solution are both unique.

Theorem 2.5.3 relaxes the condition of pair $(A, B)$ from controllability to stabilizability and establishes the connection between SCE equation system and the eigenvalues of the corresponding matrix. Actually similar to Theorem 2.5.2, it also involves the CARE. We use the stabilizing solution of the corresponding CARE to construct an invertible transformation $U$ satisfying the condition in Lemma 2.5.1. Notice that in the proof of Theorem 2.5.3, $\mathcal{A}=A-B R^{-1} B^{T} \Pi-\frac{\rho}{2} I$ corresponds to $A$ in Theorem 2.3.9; $Q_{\Gamma}$ corresponds to $-C$ and $B R^{-1} B^{T}$ corresponds to $D$. In addition, $D$ in Theorem 2.3.9 should be nonnegative definite. Since $R$ is positive definite, we can obtain that $R^{-1}$ is also positive and then $B R^{-1} B^{T}$ is nonnegative definite.

\subsection{Computational Methods}

\subsubsection{The Stabilizing Solution}

From Theorem 2.5.2, Theorem 2.5.3 and their proofs, we can find that it is quite important to find a stabilizing solution of CARE of the following form:

$$
X \mathcal{A}+\mathcal{A}^{T} X-X B R^{-1} B^{T} X-Q=0
$$

where $Q$ is symmetric and $R$ is positive definite. We will use eigenvectors and generalized eigenvectors of $H$ to compute the stabilizing solution, where

$$
H=\left(\begin{array}{cc}
\mathcal{A} & -B R^{-1} B^{T} \\
Q & -\mathcal{A}^{T}
\end{array}\right)
$$

and assume that $H$ has no purely imaginary eigenvalues. Notices that $Q$ is allowed to be indefinite.

Definition 2.6.1. Consider an eigenvalue problem

$$
H z=\lambda z
$$


$\lambda$ is an eigenvalue when $\operatorname{det}(H-\lambda I)=0$ and eigenvectors of $\lambda$ is all nonzero vectors which satisfy equation (2.42). The generalized eigenvectors are all the nonzero vectors $z$ such that $(H-\lambda I)^{r} z=0$ and $(H-\lambda I)^{r-1} z \neq 0$, where $r$ is a positive integer.

From the theory related to Jordan canonical form [20], there exists an invertible matrix $T$, such that $T^{-1} H T=S$, where $T$ consists of $2 n$ independent generalized eigenvectors and $S$ is the Jordan canonical form of $H$. According to our assumption that $H$ has no purely imaginary eigenvalues, $H$ has $n$ stable eigenvalues and $n$ unstable eigenvalues (see Proposition 2.2.2). Thus, by reordering the generalized eigenvectors, we can find an invertible $T=\left(\begin{array}{ll}U & V\end{array}\right)$, such that

$$
T^{-1} H T=\left(\begin{array}{cc}
S_{11} & 0 \\
0 & S_{22}
\end{array}\right)
$$

where $U$ consists of generalized eigenvectors corresponding to stable eigenvalues which are all in Jordan block $S_{11}$, and $V$ consists of generalized eigenvectors corresponding to unstable eigenvalues which are all in Jordan block $S_{22}$.

Now by partitioning $U$ into two $n \times n$ matrices

$$
U=\left(\begin{array}{c}
U_{1} \\
U_{2}
\end{array}\right)
$$

we obtain that

$$
H\left(\begin{array}{l}
U_{1} \\
U_{2}
\end{array}\right)=\left(\begin{array}{l}
U_{1} \\
U_{2}
\end{array}\right) S_{11}=\left(\begin{array}{l}
U_{1} S_{11} \\
U_{2} S_{11}
\end{array}\right)
$$

which implies that

$$
\begin{aligned}
\mathcal{A} U_{1}-B R^{-1} B^{T} U_{2} & =U_{1} S_{11}, \\
Q U_{1}-\mathcal{A}^{T} U_{2} & =U_{2} S_{11} .
\end{aligned}
$$

Here, if we assume that $U_{1}$ is invertible (actually we will prove it), from (2.43), we can obtain

$$
U_{1}^{-1} \mathcal{A} U_{1}-U_{1}^{-1} B R^{-1} B^{T} U_{2}=S_{11}
$$


Then combining (2.45) with (2.44), we have

$$
Q U_{1}-\mathcal{A}^{T} U_{2}=U_{2} U_{1}^{-1} \mathcal{A} U_{1}-U_{2} U_{1}^{-1} B R^{-1} B^{T} U_{2}
$$

i.e.

$$
U_{2} U_{1}^{-1} \mathcal{A}+\mathcal{A}^{T} U_{2} U_{1}^{-1}-U_{2} U_{1}^{-1} B R^{-1} B^{T} U_{2} U_{1}^{-1}-Q=0
$$

which means $U_{2} U_{1}^{-1}$ is the solution of CARE (2.41). Actually, we will prove a conclusion that since $S_{11}$ is stable, $U_{2} U_{1}^{-1}$ is the stabilizing solution of CARE (2.41).

Pappas, Laub and Sandell [17] presented a discrete-time version of Lemma 2.6.2 and Lemma 2.6.3 below for the case where the state weight matrix in their Riccati equation is nonnegative definite.

Lemma 2.6.2. If there exists a solution $X$ of CARE (2.41), $X$ must have the form $X=$ $T_{2} T_{1}^{-1}$, where $\left(\begin{array}{l}T_{1} \\ T_{2}\end{array}\right)$ consists of $n$ generalized eigenvectors of $H$.

Proof. Let $K=\mathcal{A}-B R^{-1} B^{T} X$ and then there exists an invertible $T_{1}$, such that $T_{1}^{-1} K T_{1}=$ $\hat{S}$, where $\hat{S}$ is the Jordan canonical form of $K$. Define $T_{2}=X T_{1}$. Then we have $X=T_{2} T_{1}^{-1}$. Since $K=\mathcal{A}-B R^{-1} B^{T} X$ and $T_{1}^{-1} K T_{1}=\hat{S}$, we have

$$
\mathcal{A}-B R^{-1} B^{T} X=T_{1} \hat{S} T_{1}^{-1}
$$

and then

$$
\mathcal{A} T_{1}-B R^{-1} B^{T} X T_{1}=T_{1} \hat{S}
$$

Finally we obtain

$$
\mathcal{A} T_{1}-B R^{-1} B^{T} T_{2}=T_{1} \hat{S}
$$

From CARE (2.41), we have

$$
\mathcal{A}^{T} X+X K-Q=0
$$

Thus, we have

$$
\mathcal{A}^{T} X+X T_{1} \hat{S} T_{1}^{-1}-Q=0
$$


and then

$$
Q-\mathcal{A}^{T} T_{2}=T_{2} \hat{S}
$$

From (2.47) and (2.48), we have

$$
\begin{aligned}
\left(\begin{array}{cc}
\mathcal{A} & -B R^{-1} B^{T} \\
Q & -\mathcal{A}^{T}
\end{array}\right)\left(\begin{array}{l}
T_{1} \\
T_{2}
\end{array}\right) & =\left(\begin{array}{c}
\mathcal{A} T_{1}-B R^{-1} B^{T} T_{2} \\
Q-\mathcal{A}^{T} T_{2}
\end{array}\right)=\left(\begin{array}{c}
T_{1} \hat{S} \\
T_{2} \hat{S}
\end{array}\right) \\
& =\left(\begin{array}{c}
T_{1} \\
T_{2}
\end{array}\right) \hat{S} .
\end{aligned}
$$

Thus, $\left(\begin{array}{c}T_{1} \\ T_{2}\end{array}\right)$ is $n$ generalized eigenvectors of $H$.

Lemma 2.6.3. Consider $\left(\begin{array}{l}V_{1} \\ V_{2}\end{array}\right)$ consisting of $n$ generalized eigenvectors of $H$ and corresponding to the Jordan canonical block $\hat{S}$. Assume that $V_{1}$ is invertible. Then

1) $X=V_{2} V_{1}^{-1}$ is a real solution of CARE (2.41).

2) $X=V_{2} V_{1}^{-1}$ is the stabilizing solution CARE (2.41) if and only if $\hat{S}$ is stable.

Proof. Our method follows [18].

1) Similar to (2.49), we have

$$
H\left(\begin{array}{l}
V_{1} \\
V_{2}
\end{array}\right)=\left(\begin{array}{l}
V_{1} \\
V_{2}
\end{array}\right) \hat{S}=\left(\begin{array}{c}
V_{1} \hat{S} \\
V_{2} \hat{S}
\end{array}\right)
$$

which implies that:

$$
\begin{aligned}
\mathcal{A} V_{1}-B R^{-1} B^{T} V_{2} & =V_{1} \hat{S}, \\
Q V_{1}-\mathcal{A}^{T} V_{2} & =V_{2} \hat{S} .
\end{aligned}
$$

Since $V_{1}$ is invertible, we have

$$
V_{1}^{-1} \mathcal{A} V_{1}-V_{1}^{-1} B R^{-1} B^{T} V_{2}=\hat{S} .
$$


Then combining (2.53) with (2.52), we have

$$
Q V_{1}-\mathcal{A}^{T} V_{2}=V_{2} V_{1}^{-1} \mathcal{A} V_{1}-V_{2} V_{1}^{-1} B R^{-1} B^{T} V_{2},
$$

i.e.

$$
V_{2} V_{1}^{-1} \mathcal{A}+\mathcal{A}^{T} V_{2} V_{1}^{-1}-V_{2} V_{1}^{-1} B R^{-1} B^{T} V_{2} V_{1}^{-1}-Q=0 .
$$

Thus, $X=V_{2} V_{1}^{-1}$ is the solution of CARE (2.41).

2) From (2.50), we have

$$
\left(\begin{array}{l}
V_{1} \\
V_{2}
\end{array}\right)^{T}\left(\begin{array}{cc}
0 & I \\
-I & 0
\end{array}\right) H\left(\begin{array}{l}
V_{1} \\
V_{2}
\end{array}\right)=\left(\begin{array}{l}
V_{1} \\
V_{2}
\end{array}\right)^{T}\left(\begin{array}{cc}
0 & I \\
-I & 0
\end{array}\right)\left(\begin{array}{l}
V_{1} \\
V_{2}
\end{array}\right) \hat{S} .
$$

Since $H$ is a Hamiltonian matrix, the left-hand side of (2.54) is symmetric. Thus, the RHS of (2.54) is also symmetric and then we obtain

$$
\hat{S}^{T}\left(\begin{array}{l}
V_{1} \\
V_{2}
\end{array}\right)^{T}\left(\begin{array}{cc}
0 & -I \\
I & 0
\end{array}\right)\left(\begin{array}{l}
V_{1} \\
V_{2}
\end{array}\right)=\left(\begin{array}{c}
V_{1} \\
V_{2}
\end{array}\right)^{T}\left(\begin{array}{cc}
0 & I \\
-I & 0
\end{array}\right)\left(\begin{array}{l}
V_{1} \\
V_{2}
\end{array}\right) \hat{S}
$$

i.e.

$$
\left(V_{1}^{T} V_{2}-V_{2}^{T} V_{1}\right) \hat{S}+\hat{S}^{T}\left(V_{1}^{T} V_{2}-V_{2}^{T} V_{1}\right)=0
$$

Denote $V_{1}^{T} V_{2}-V_{2}^{T} V_{1}$ as $Y$. Equation (2.55) is

$$
Y \hat{S}+\hat{S} Y=0
$$

which is a Lyapunov equation of $Y$. If $\hat{S}$ is stable, this Lyapunov equation has a unique solution $Y=0$. Thus, $V_{1}^{T} V_{2}=V_{2}^{T} V_{1}$, and then $V_{2} V_{1}^{-1}=\left(V_{1}^{T}\right)^{-1} V_{2}^{T}=\left(V_{2} V_{1}^{-1}\right)^{T}$, i.e. $X=V_{2} V_{1}^{-1}$ is symmetric. Since we have

$$
\mathcal{A} V_{1}-B R^{-1} B^{T} V_{2}=V_{1} \hat{S}
$$

so

$$
\mathcal{A}-B R^{-1} B^{T} X=V_{1} \hat{S} V_{1}^{-1}
$$


We have $V_{1} \hat{S} V_{1}^{-1}$ is stable. Thus $\mathcal{A}-B R^{-1} B^{T} X$ is stable, which means $X$ is the stabilizing solution.

If $X$ is the stabilizing solution, then $\mathcal{A}-B R^{-1} B^{T} X$ is stable. Thus, $V_{1} \hat{S} V_{1}^{-1}$ is stable, which implies $\hat{S}$ is stable.

Theorem 2.6.4. Assume $(\mathcal{A}, B)$ is stabilizable and the Hamiltonian matrix $H$ has no purely imaginary eigenvalues. Then $U_{1}$ is invertible and $X=U_{2} U_{1}^{-1}$ is the stabilizing solution of CARE (2.41), where $\left(\begin{array}{l}U_{1} \\ U_{2}\end{array}\right)$ consists of $n$ generalized eigenvectors of $H$ and corresponds to the stable Jordan canonical block $S_{11}$.

Proof. According to Theorem 2.3.9 and Theorem 2.3.12, there exists a unique stabilizing solution of CARE (2.41), denoted by $X_{+}$. By Lemma 2.6.2, $X_{+}$must have the form $X_{+}=$ $V_{2} V_{1}^{-1}$, where $\left(\begin{array}{l}V_{1} \\ V_{2}\end{array}\right)$ consists of $n$ generalized eigenvectors of $H$, and the corresponding Jordan canonical block is $\hat{S}$. By Lemma 2.6.3, $\hat{S}$ is stable. Since $S_{11}$ and $\hat{S}$ are both stable, they both includes all the stable eigenvalues of $H$. Thus, there exists a orthogonal matrix $T$, such that $T^{-1} S_{11} T=\hat{S}$. In addition, we have $\left(\begin{array}{c}U_{1} \\ U_{2}\end{array}\right)=\left(\begin{array}{ll}T & 0 \\ 0 & I\end{array}\right)\left(\begin{array}{l}V_{1} \\ V_{2}\end{array}\right)$. Thus, $U_{1}=T V_{1}$ is invertible and then by Lemma 2.6.3, $X=U_{2} U_{1}^{-1}$ is the stabilizing solution of CARE $(2.41)$.

Theorem 2.6.4 guarantees that when we find a set of generalized eigenvectors corresponding to the stable Jordan canonical block of $H$, the stabilizing solution can be found by this set of generalized eigenvectors. In addition, we just require two assumptions that $(\mathcal{A}, B)$ is stabilizing and $H$ has no purely imaginary eigenvalues. Indeed, these two assumptions are to guarantee the existence and uniqueness of the stabilizing solution. On the other hand, a similar idea is presented in [17]. When $Q$ is nonnegative definite, Pappas, Laub and Sandell [17] used generalized eigenvectors to find the positive definite solution of a discrete-time algebraic Riccati equation. In our assumption, $Q$ only needs to be symmetric.

However, computing generalized eigenvectors is still complicated. Now we introduce a more convenient method to compute the stabilizing solution of CARE (2.41). Here, we still assume $(\mathcal{A}, B)$ is stabilizable and $H$ has no purely imaginary eigenvalues. 
Theorem 2.6.5. [12] There exists an orthogonal transformation $V \in \mathbb{R}^{n \times n}$, such that

$$
V^{T} H V=\left(\begin{array}{cc}
H_{11} & H_{12} \\
0 & H_{22}
\end{array}\right)
$$

where all eigenvalues of $H_{11}$ are the stable eigenvalues of $H$.

We refer to $\left(\begin{array}{cc}H_{11} & H_{12} \\ 0 & H_{22}\end{array}\right)$ as the real Schur form and $V$ consists of $2 n$ independent vectors which are called Schur vectors. If we partition $V$ into four $n \times n$ block $\left(\begin{array}{ll}V_{11} & V_{12} \\ V_{21} & V_{22}\end{array}\right)$, $\left(\begin{array}{l}V_{11} \\ V_{21}\end{array}\right)$ consists of $n$ Schur vectors corresponding to stable Schur block $H_{11}$.

Lemma 2.6.6. $\left[12, p\right.$. 915] Denote $\left(\begin{array}{l}V_{11} \\ V_{21}\end{array}\right)$ as the Schur vectors corresponding to the stable Schur block of $H$ and $\left(\begin{array}{l}U_{1} \\ U_{2}\end{array}\right)$ as the generalized vectors corresponding to the stable Jordan canonical block of $H$, there exists an invertible $T \in \mathbb{R}^{n \times n}$ such that

$$
\left(\begin{array}{c}
V_{11} \\
V_{21}
\end{array}\right)=\left(\begin{array}{c}
U_{1} \\
U_{2}
\end{array}\right) T
$$

The proof of Lemma 2.6.6 is presented in [12], which corresponds to [12, Remark 1, p915]. This lemma establishes the connection between Schur vectors and generalized eigenvectors, so we can extend the conclusion about generalized eigenvectors to the Schur vectors.

Theorem 2.6.7. Assume $(\mathcal{A}, B)$ is stabilizable and the Hamiltonian matrix $H$ has no purely imaginary eigenvalues. Then $V_{11}$ is invertible and $X=V_{21} V_{11}^{-1}$ is the stabilizing solution of CARE (2.41), where $\left(\begin{array}{l}V_{1} \\ V_{2}\end{array}\right)$ consists of $n$ Schur vectors of $H$ and corresponds to the stable Schur block $H_{11}$.

Proof. By Theorem 2.6.4, there exists a set of generalized eigenvectors corresponding to stable Jordan block of $H$, such that $U_{2} U_{1}^{-1}$ is the stabilizing solution of CARE (2.41).

By Lemma 2.6.6, we have $\left(\begin{array}{c}V_{11} \\ V_{21}\end{array}\right)=\left(\begin{array}{c}U_{1} \\ U_{2}\end{array}\right) T$, so $V_{11}=U_{1} T$ is invertible. Furthermore, $X=V_{21} V_{11}^{-1}=\left(U_{2} T\right)\left(U_{1} T\right)^{-1}=U_{2} T T^{-1} U_{1}^{-1}=U_{2} U_{1}^{-1}$ is a stabilizing solution. 
Now we show some examples to compute the stabilizing solution of the following CARE (2.56) and the results will be used in further discussions. All the computation results are based on Matlab.

$$
X \mathcal{A}+\mathcal{A}^{T} X-X B R^{-1} B^{T} X-Q_{\Gamma}=0
$$

where $\mathcal{A}=A-B R^{-1} B^{T} \Pi-\frac{\rho}{2} I, Q_{\Gamma}=\Gamma^{T} Q+Q \Gamma-\Gamma^{T} Q \Gamma$ and the corresponding Hamiltonian matrix is

$$
H=\left(\begin{array}{cc}
\mathcal{A} & -B R^{-1} B^{T} \\
Q_{\Gamma} & \mathcal{A}^{T}
\end{array}\right)
$$

In the examples below, we specify the parameters, including the matrix $A$, which further determines $\mathcal{A}$.

Example 2.6.8. Consider a scalar example where $A=2, B=1, Q=2, R=1, \rho=1, \Gamma=1$. We have

$$
H=\left(\begin{array}{cc}
\mathcal{A} & -B R^{-1} B^{T} \\
Q_{\Gamma} & \mathcal{A}^{T}
\end{array}\right)=\left(\begin{array}{cc}
-2.0616 & -1.0000 \\
2.0000 & 2.0616
\end{array}\right)
$$

and the eigenvalues of $H$ is -1.5 and 1.5 , so $H$ has no purely imaginary eigenvalues. The Schur decomposition is as follow:

$$
H=\left(\begin{array}{cc}
-0.8719 & -0.4896 \\
0.4896 & -0.8719
\end{array}\right)\left(\begin{array}{cc}
-1.5000 & -3.0000 \\
0 & 1.5000
\end{array}\right)\left(\begin{array}{cc}
-0.8719 & -0.4896 \\
0.4896 & -0.8719
\end{array}\right)^{-1}
$$

so $V_{11}=-0.8719, V_{21}=0.4896$ and then $X=V_{21} V_{11}^{-1}=-0.5616$.

Example 2.6.8 shows that a stabilizing solution $X$ may be negative (definite).

Example 2.6.9. Consider $A=\left(\begin{array}{cc}1 & -1 \\ 0 & 2\end{array}\right), B=\left(\begin{array}{l}1 \\ 1\end{array}\right), Q=\left(\begin{array}{ll}1 & 0 \\ 0 & 1\end{array}\right), \Gamma=\left(\begin{array}{cc}1 & 0 \\ 0.5 & 1\end{array}\right)$ and $\rho=1, R=1$.

We have

$$
H=\left(\begin{array}{cccc}
2.7370 & -8.3699 & -1.0000 & -1.0000 \\
2.2370 & -5.8699 & -1.0000 & -1.0000 \\
-1.0000 & -1.0000 & -2.7370 & -2.2370 \\
-1.0000 & 0 & 8.3699 & 5.8699
\end{array}\right)
$$


and the eigenvalues of $H$ are $\pm 1.1901 \pm 0.2034 i$, so $H$ has no purely imaginary eigenvalues. The Schur decomposition is as follow:

$$
H=V\left(\begin{array}{cccc}
-1.1901 & 2.7201 & -0.0793 & -8.4223 \\
-0.0152 & -1.1901 & -12.1723 & 0.0793 \\
0 & 0 & 1.1901 & 2.7201 \\
0 & 0 & -0.0152 & 1.1901
\end{array}\right) V^{-1}
$$

where

$$
V=\left(\begin{array}{cccc}
0.6136 & -0.5467 & 0.2808 & -0.4958 \\
0.2808 & -0.4957 & -0.6137 & 0.5466 \\
-0.4958 & -0.2808 & -0.5467 & -0.6136 \\
0.5466 & 0.6137 & -0.4957 & -0.2808
\end{array}\right) .
$$

Thus, $V_{11}=\left(\begin{array}{cc}0.6136 & -0.5467 \\ 0.2808 & -0.4957\end{array}\right), V_{21}=\left(\begin{array}{cc}-0.4958 & -0.2808 \\ 0.5466 & 0.6137\end{array}\right)$ and $X=V_{21} V_{11}^{-1}=$ $\left(\begin{array}{cc}-2.1548 & 2.9428 \\ 2.9428 & -4.4836\end{array}\right)$. Actually, we can check that $\mathcal{A}-B R^{-1} B^{T} X=\left(\begin{array}{cc}1.9489 & -6.8292 \\ 1.4489 & -4.3292\end{array}\right)$, so its eigenvalues are $-1.1901 \pm 0.2034 i$. Thus, $X$ is a stabilizing solution, which means that our computational method works.

\subsubsection{Computational Results}

Now recall our methods of finding the unique solution $(\bar{x}, s) \in C_{\rho / 2}\left([0, \infty), \mathbb{R}^{2 n}\right)$. Our original ODE system has the following form:

$$
\left(\begin{array}{c}
\frac{d \bar{x}}{d t} \\
\frac{d s}{d t}
\end{array}\right)=\left(\begin{array}{cc}
A-B R^{-1} B^{T} \Pi & -B R^{-1} B^{T} \\
Q_{\Gamma} & \rho I-A^{T}+\Pi B R^{-1} B^{T}
\end{array}\right)\left(\begin{array}{c}
\bar{x} \\
s
\end{array}\right)+\left(\begin{array}{c}
0 \\
\eta_{\Gamma}
\end{array}\right)
$$

By the transformation matrix $T=\left(\begin{array}{cc}e^{\frac{\rho}{2} t} & 0 \\ 0 & e^{\frac{\rho}{2} t}\end{array}\right)$, we obtain a new ODE system of $(\tilde{x}, \tilde{s})$ :

$$
\left(\begin{array}{c}
\frac{d \tilde{x}}{d t} \\
\frac{d \tilde{s}}{d t}
\end{array}\right)=\left(\begin{array}{cc}
A-B R^{-1} B^{T} \Pi-\frac{\rho}{2} I & -B R^{-1} B^{T} \\
Q_{\Gamma} & \frac{\rho}{2} I-A^{T}+\Pi B R^{-1} B^{T}
\end{array}\right)\left(\begin{array}{c}
\tilde{x} \\
\tilde{s}
\end{array}\right)+\left(\begin{array}{c}
0 \\
e^{-\frac{\rho}{2} t} \eta_{\Gamma}
\end{array}\right)
$$


By selecting a transformation matrix $U=\left(\begin{array}{cc}I & 0 \\ X_{+} & I\end{array}\right)$, we obtain the ODE system of $(\hat{x}, \hat{s})$ :

$$
\left(\begin{array}{c}
\frac{d \hat{x}}{d t} \\
\frac{d \hat{s}}{d t}
\end{array}\right)=\left(\begin{array}{cc}
\mathcal{A}_{C}^{T} & -B R^{-1} B^{T} \\
0 & -\mathcal{A}_{C}^{T}
\end{array}\right)\left(\begin{array}{l}
\hat{x} \\
\hat{s}
\end{array}\right)+\left(\begin{array}{c}
0 \\
e^{-\frac{\rho}{2} t} \eta_{\Gamma}
\end{array}\right)
$$

where $\mathcal{A}_{C}=\mathcal{A}-B R^{-1} B^{T} X_{+}$. Since

$$
\left(\begin{array}{l}
\hat{x} \\
\hat{s}
\end{array}\right)=U^{-1}\left(\begin{array}{l}
\tilde{x} \\
\tilde{s}
\end{array}\right)
$$

and

$$
\left(\begin{array}{c}
\tilde{x} \\
\tilde{s}
\end{array}\right)=T^{-1}\left(\begin{array}{l}
\bar{x} \\
s
\end{array}\right)
$$

we obtain that

$$
\left(\begin{array}{l}
\bar{x}(t) \\
s(t)
\end{array}\right)=T U\left(\begin{array}{l}
\hat{x}(t) \\
\hat{s}(t)
\end{array}\right)=\left(\begin{array}{cc}
e^{\frac{\rho}{2} t} I & 0 \\
e^{\frac{\rho}{2} t} X_{+} & e^{\frac{\rho}{2} t} I
\end{array}\right)\left(\begin{array}{c}
\hat{x}(t) \\
\hat{s}(t)
\end{array}\right)=\left(\begin{array}{c}
e^{\frac{\rho}{2} t} \hat{x}(t) \\
X_{+} e^{\frac{\rho}{2} t} \hat{x}(t)+e^{\frac{\rho}{2} t} \hat{s}(t)
\end{array}\right)
$$

Here $X_{+}$is the stabilizing solution of the following CARE:

$$
X B R^{-1} B^{T} X-X \mathcal{A}-\mathcal{A}^{T} X+Q_{\Gamma}=0,
$$

where $\mathcal{A}=A-B R^{-1} B^{T} \Pi-\frac{\rho}{2} I$.

According to Theorem 2.5.3, for obtaining the solution $(\bar{x}, s)$ of SCE equation system (2.57), such that $(\bar{x}, s) \in C_{\rho / 2}\left([0, \infty), \mathbb{R}^{2 n}\right)$, we (must) select the initial condition

$$
s_{0}=X_{+} \bar{x}_{0}-\int_{0}^{+\infty} e^{\left(\mathcal{A}_{C}^{T}-\frac{\rho}{2} I\right) \tau} \eta_{\Gamma} d \tau
$$

where $\mathcal{A}_{C}^{T}=\left(\mathcal{A}-B R^{-1} B^{T} X_{+}\right)^{T}$. We can firstly find the solution $(\tilde{x}, \tilde{s})$ of ODE system (2.58) under the initial condition $\hat{s}_{0}=-\int_{0}^{+\infty} e^{\mathcal{A}_{C}^{T} \tau} \eta_{\Gamma} e^{-\rho \tau / 2} d \tau$ and then use (2.61) to obtain the corresponding solution of SCE equation system (2.57). Indeed, we can also solve the SCE equations system (2.57) directly under the initial condition $s_{0}$. Here is a summary of our computational method: 


\begin{tabular}{|c|c|}
\hline Step 1 & Check the eigenvalues of the Hamiltonian matrix $H$ \\
\hline Step 2 & Obtain the Schur vectors by Schur decomposition \\
\hline Step 3 & Compute the stabilizing solution to the corresponding CARE \\
\hline Step 4 & Compute the initial condition $s_{0}$ \\
\hline Step 5 & Compute the solution to the SCE equation system \\
\hline
\end{tabular}

Example 2.6.10. Consider a scalar example: $A=2, B=1, Q=2, R=1, \eta=1, \rho=1, \Gamma=\gamma$ and the initial state $x_{0}=1$. The SCE equation system is

$$
\left(\begin{array}{l}
\frac{d \bar{x}}{d t} \\
\frac{d s}{d t}
\end{array}\right)=\left(\begin{array}{cc}
-1.5616 & -1.0000 \\
2\left(2 \gamma-\gamma^{2}\right) & 2.5616
\end{array}\right)\left(\begin{array}{l}
\bar{x} \\
s
\end{array}\right)+\left(\begin{array}{c}
0 \\
2(1-\gamma)
\end{array}\right)
$$

Now, we consider some special cases.

1) For $\gamma=1$, we have $Q_{\Gamma}=Q>0$. The SCE equation system is

$$
\left(\begin{array}{l}
\frac{d \bar{x}}{d t} \\
\frac{d s}{d t}
\end{array}\right)=\left(\begin{array}{cc}
-1.5616 & -1.0000 \\
2 & 2.5616
\end{array}\right)\left(\begin{array}{l}
\bar{x} \\
s
\end{array}\right)
$$

and

$$
H=\left(\begin{array}{cc}
A-B R^{-1} B^{T} \Pi-\frac{\rho}{2} I & -B R^{-1} B^{T} \\
Q_{\Gamma} & \frac{\rho}{2} I-A^{T}+\Pi B R^{-1} B^{T}
\end{array}\right)=\left(\begin{array}{cc}
-2.0616 & -1.0000 \\
2.0000 & 2.0616
\end{array}\right)
$$

The eigenvalues of $H$ is -1.5 and 1.5, so $H$ has no purely imaginary eigenvalues. By solving the CARE (2.62), $X_{+}=-0.5615$ and then $\mathcal{A}_{C}=-1.5$.

Thus, we select the initial condition $s_{0}=X_{+} \bar{x}_{0}-\int_{0}^{+\infty} e^{\left(\mathcal{A}_{C}^{T}-\frac{\rho}{2} I\right) \tau} \eta_{\Gamma} d \tau=-0.5615$. By solving the SCE equation system under the initial condition $(1,-0.5615)$, we obtain the solution $(\bar{x}(t), s(t))=\left(1.0 * e^{-1.0 t},-0.561553 * e^{-1.0 * t}\right)$ and obviously $(\bar{x}(t), s(t) \in$ $C_{1 / 2}\left([0, \infty), \mathbb{R}^{2}\right)$.

2) For $\gamma=2$, we have $Q_{\Gamma}=0$. The SCE equation system is

$$
\left(\begin{array}{c}
\frac{d \bar{x}}{d t} \\
\frac{d s}{d t}
\end{array}\right)=\left(\begin{array}{cc}
-1.5616 & -1.0000 \\
0 & 2.5616
\end{array}\right)\left(\begin{array}{l}
\bar{x} \\
s
\end{array}\right)+\left(\begin{array}{c}
0 \\
-2
\end{array}\right)
$$

and

$$
H=\left(\begin{array}{cc}
A-B R^{-1} B^{T} \Pi-\frac{\rho}{2} I & -B R^{-1} B^{T} \\
Q_{\Gamma} & \frac{\rho}{2} I-A^{T}+\Pi B R^{-1} B^{T}
\end{array}\right)=\left(\begin{array}{cc}
-2.0616 & -1.0000 \\
0 & 2.0616
\end{array}\right)
$$




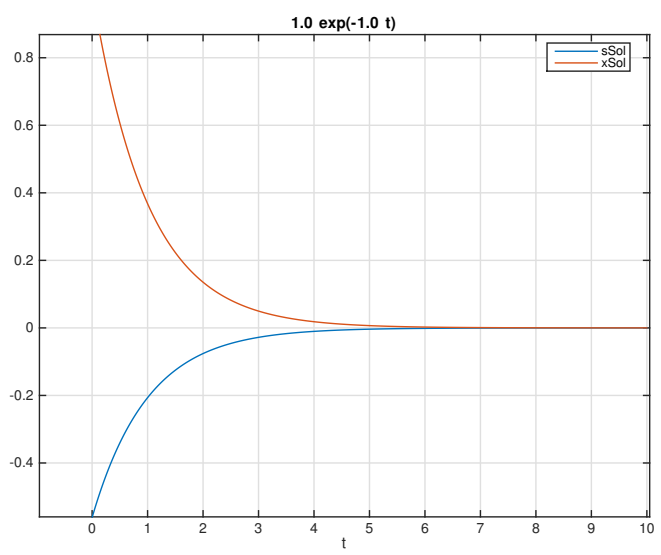

Figure 2.1: Solution when $\gamma=1$

The eigenvalues of $H$ are -2.0616 and 2.0616, so $H$ has no purely imaginary eigenvalues. Obviously the stabilizing solution $X_{+}=0$ and the initial condition we choose is $s_{0}=$ $-\int_{0}^{+\infty} e^{\left(\mathcal{A}_{C}^{T}-\frac{\rho}{2} I\right) \tau} \eta_{\Gamma} d \tau=-0.780776$. Then the corresponding solution $(\bar{x}(t), s(t))=(1.5 *$ $\left.e^{-1.56155 * t}-0.5,0.780776\right)$, where $s(t)$ is a constant.

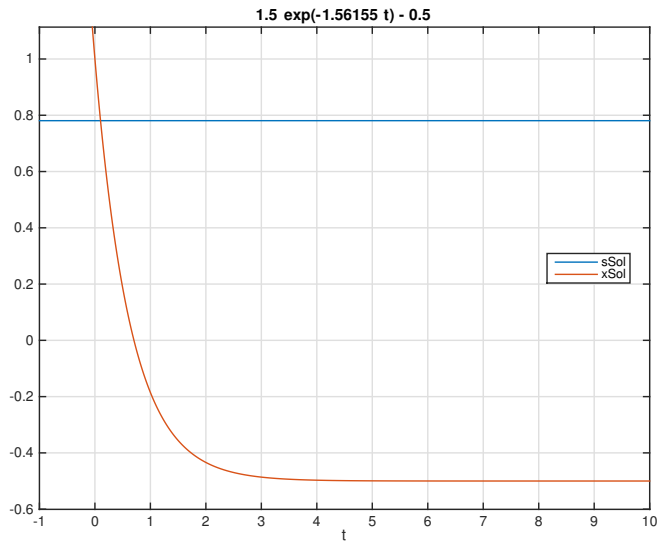

Figure 2.2: Solution when $\gamma=2$

3) For $\gamma=3$, we have $Q_{\Gamma}=-3 Q<0$.

The SCE equation system is

$$
\left(\begin{array}{c}
\frac{d \bar{x}}{d t} \\
\frac{d s}{d t}
\end{array}\right)=\left(\begin{array}{cc}
-1.5616 & -1.0000 \\
-6.0000 & 2.5616
\end{array}\right)\left(\begin{array}{l}
\bar{x} \\
s
\end{array}\right)+\left(\begin{array}{c}
0 \\
-4
\end{array}\right),
$$


and

$$
H=\left(\begin{array}{cc}
A-B R^{-1} B^{T} \Pi-\frac{\rho}{2} I & -B R^{-1} B^{T} \\
Q_{\Gamma} & \frac{\rho}{2} I-A^{T}+\Pi B R^{-1} B^{T}
\end{array}\right)=\left(\begin{array}{cc}
-2.0616 & -1.0000 \\
-6 & 2.0616
\end{array}\right) .
$$

The eigenvalues of $H$ are -3.2016 and 3.2016, so $H$ has no purely imaginary eigenvalues. By solving the CARE (2.62), $X_{+}=1.1400$ and then $\mathcal{A}_{C}=-3.2016$.

Thus, we select the initial condition $s_{0}=X_{+} \bar{x}_{0}-\int_{0}^{+\infty} e^{\left(\mathcal{A}_{C}^{T}-\frac{\rho}{2} I\right) \tau} \eta_{\Gamma} d \tau=2.22063$. By solving the SCE equation system under the initial condition $(1,2.22063)$, we obtain the solution $(\bar{x}(t), s(t))=\left(1.4 e^{-2.70156 t}-0.4,1.59601 e^{-2.70156 t}+0.624621\right)$ and obviously $\left(\bar{x}(t), s(t) \in C_{1 / 2}\left([0, \infty), \mathbb{R}^{2}\right)\right.$.

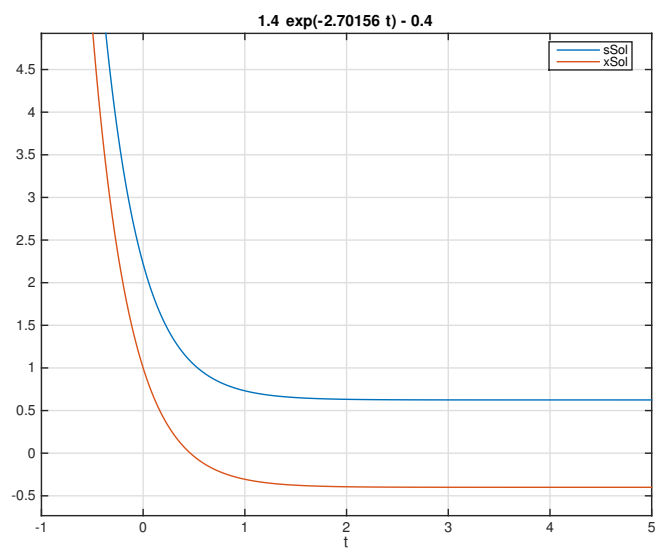

Figure 2.3: Solution when $\gamma=3$

Example 2.6.10 shows that our method works well when $Q_{\Gamma}$ is positive, negative and zero. Thus, we can handle the situation that $Q_{\Gamma}>0$. This situation is not covered by [8, Theorem 4.3]. However, if $Q_{\Gamma}$ is not a scalar, we should consider a situation that $Q_{\Gamma}$ is an indefinite matrix.

Example 2.6.11. Consider $A=\left(\begin{array}{cc}1 & -1 \\ 0 & 2\end{array}\right), B=\left(\begin{array}{l}1 \\ 1\end{array}\right), Q=\left(\begin{array}{ll}1 & 0 \\ 0 & 1\end{array}\right), \eta=\left(\begin{array}{l}1 \\ 0\end{array}\right), \Gamma=$ $\gamma\left(\begin{array}{cc}1 & 0 \\ 0.5 & 1\end{array}\right), \rho=1, R=1$, and the initial state is $x_{0}=\left(\begin{array}{l}1 \\ 1\end{array}\right)$.

1) For $\gamma=2$, we have $Q_{\Gamma}=\left(\begin{array}{cc}-1 & -1 \\ -1 & 0\end{array}\right)$. Since the eigenvalues of $Q_{\Gamma}$ are -1.618 and $0.618, Q_{\Gamma}$ is an indefinite matrix. 
The SCE equation system is

$$
\left(\begin{array}{c}
\frac{d \bar{x}_{1}}{d t} \\
\frac{d \bar{x}_{2}}{d t} \\
\frac{d s_{1}}{d t} \\
\frac{d s_{2}}{d t}
\end{array}\right)=\left(\begin{array}{cccc}
3.2370 & -8.3699 & -1.0000 & -1.0000 \\
2.2370 & -5.3699 & -1.0000 & -1.0000 \\
-1.0000 & -1.0000 & -2.2370 & -2.2370 \\
-1.0000 & 0 & 8.3699 & 6.3699
\end{array}\right)\left(\begin{array}{c}
\bar{x}_{1} \\
\bar{x}_{2} \\
s_{1} \\
s_{2}
\end{array}\right)+\left(\begin{array}{c}
0 \\
0 \\
-1 \\
0
\end{array}\right)
$$

and

$$
\begin{aligned}
H & =\left(\begin{array}{cccc}
A-B R^{-1} B^{T} \Pi-\frac{\rho}{2} I & -B R^{-1} B^{T} \\
Q_{\Gamma} & \frac{\rho}{2} I-A^{T}+\Pi B R^{-1} B^{T}
\end{array}\right) \\
& =\left(\begin{array}{cccc}
2.7370 & -8.3699 & -1.0000 & -1.0000 \\
2.2370 & -5.8699 & -1.0000 & -1.0000 \\
-1.0000 & -1.0000 & -2.7370 & -2.2370 \\
-1.0000 & 0 & 8.3699 & 5.8699
\end{array}\right) .
\end{aligned}
$$

The eigenvalues of $H$ are $-1.9655 \pm 0.3365 i$ and $1.9655 \pm 0.3365 i$, so $H$ has no purely imaginary eigenvalues. By solving the CARE (2.62), $X_{+}=\left(\begin{array}{cc}3.5811 & -4.4401 \\ -4.4401 & 6.0972\end{array}\right)$ and then $\mathcal{A}_{C}=\left(\begin{array}{cc}3.5960 & -10.0270 \\ 3.0960 & -7.5270\end{array}\right)$

Thus, we select the initial condition $s_{0}=X_{+} \bar{x}_{0}-\int_{0}^{+\infty} e^{\left(\mathcal{A}_{C}^{T}-\frac{\rho}{2} I\right) \tau} \eta_{\Gamma} d \tau=\left(\begin{array}{c}0.437344 \\ 0.0377865\end{array}\right)$. By solving the SCE equation system under the initial condition $(1,1,0.437344,0.0377865)$, we obtain the corresponding solution. From Figure 2.4, obviously $\left(\bar{x}_{1}(t), \bar{x}_{2}(t), s_{1}(t), s_{2}(t)\right) \in$ $C_{1 / 2}\left([0, \infty), \mathbb{R}^{4}\right)$.

2) For $\gamma=1$, we have $Q_{\Gamma}=\left(\begin{array}{cc}0.7500 & 0 \\ 0 & 1.0000\end{array}\right)$. Since the eigenvalues of $Q_{\Gamma}$ are 0.7500 and $1, Q_{\Gamma}$ is a positive definite matrix.

The SCE equation system is

$$
\left(\begin{array}{c}
\frac{d \bar{x}_{1}}{d t} \\
\frac{d \bar{x}_{2}}{d t} \\
\frac{d s_{1}}{d t} \\
\frac{d s_{2}}{d t}
\end{array}\right)=\left(\begin{array}{cccc}
3.2370 & -8.3699 & -1.0000 & -1.0000 \\
2.2370 & -5.3699 & -1.0000 & -1.0000 \\
0.7500 & 0 & -2.2370 & -2.2370 \\
0 & 1 & 8.3699 & 6.3699
\end{array}\right)\left(\begin{array}{l}
\bar{x}_{1} \\
\bar{x}_{2} \\
s_{1} \\
s_{2}
\end{array}\right)
$$




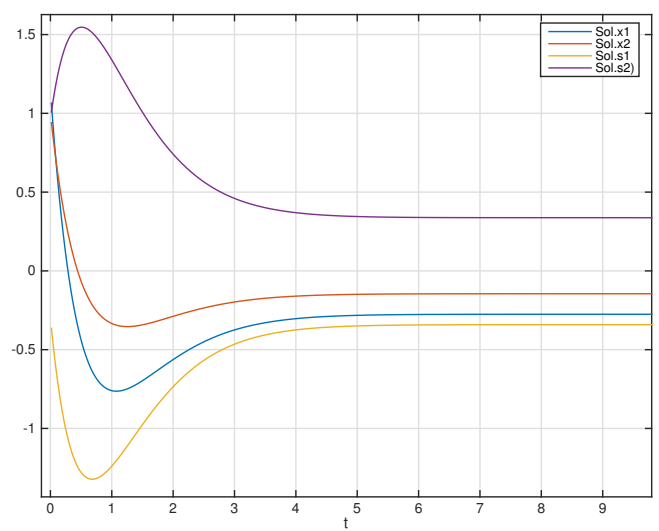

Figure 2.4: Solution when $\gamma=2$

and

$$
\begin{aligned}
H & =\left(\begin{array}{cccc}
A-B R^{-1} B^{T} \Pi-\frac{\rho}{2} I & -B R^{-1} B^{T} \\
Q_{\Gamma} & \frac{\rho}{2} I-A^{T}+\Pi B R^{-1} B^{T}
\end{array}\right) \\
& =\left(\begin{array}{cccc}
2.7370 & -8.3699 & -1.0000 & -1.0000 \\
2.2370 & -5.8699 & -1.0000 & -1.0000 \\
-1.0000 & -1.0000 & -2.7370 & -2.2370 \\
-1.0000 & 0 & 8.3699 & 5.8699
\end{array}\right) .
\end{aligned}
$$

The eigenvalues of $H$ are $-1.1901 \pm 0.2034 i$ and $1.1901 \pm 0.2034 i$, so $H$ has no purely imaginary eigenvalues. By solving the CARE (2.62), $X_{+}=\left(\begin{array}{cc}-2.1548 & 2.9428 \\ 2.9428 & -4.4836\end{array}\right)$.

Thus, we select the initial condition $s_{0}=X_{+} \bar{x}_{0}=\left(\begin{array}{c}0.788068 \\ -1.54074\end{array}\right)$. By solving the SCE equation system under the initial condition $(1,1,0.788068,-1.54074)$, we obtain the corresponding solution. From Figure 2.5, obviously $\left(\bar{x}_{1}(t), \bar{x}_{2}(t), s_{1}(t), s_{2}(t)\right) \in C_{1 / 2}\left([0, \infty), \mathbb{R}^{4}\right)$.

\subsubsection{Comparison}

In this section, we introduce a fixed point method, which is used to solve the SCE equation system and the Hamiltonian ODE system in [7] and [15]. Since our purpose is to compare the fixed point method with our method, we will omit some assumptions and details related to the fixed point method.

Consider this Hamiltonian ODE: 


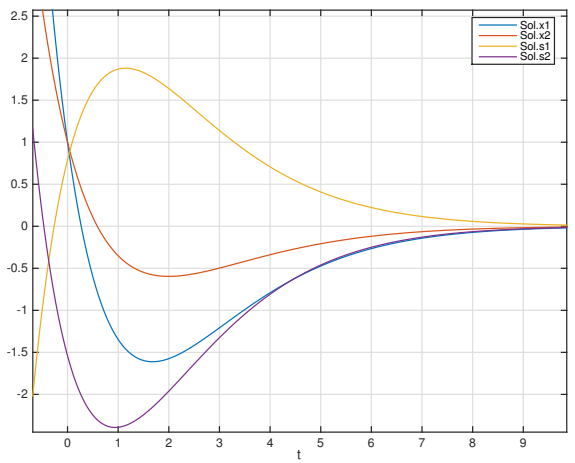

Figure 2.5: Solution when $\gamma=1$

$$
\left(\begin{array}{c}
\frac{d \tilde{x}}{d t} \\
\frac{d \tilde{s}}{d t}
\end{array}\right)=\left(\begin{array}{cc}
\mathcal{A} & -B R^{-1} B^{T} \\
Q_{\Gamma} & -\mathcal{A}^{T}
\end{array}\right)\left(\begin{array}{c}
\tilde{x} \\
\tilde{s}
\end{array}\right)+\left(\begin{array}{c}
0 \\
\tilde{\eta}_{\Gamma}
\end{array}\right),
$$

where $x(0)=x_{0}$ and the value of the initial condition $\tilde{s}_{0}$ is to be determined according to Section 2.1. Under some reasonable assumptions $[7,15]$, the solution $\tilde{x}(t)$ can be solved by fixed point equation $x(t)=[\Psi x](t)$, where

$$
\Psi(x(t))=e^{\mathcal{A} t} x_{0}+\int_{0}^{t} e^{\mathcal{A}(t-s)} B R^{-1} B^{T}\left[\int_{s}^{\infty} e^{-\mathcal{A}^{T}(s-\tau)}\left(Q_{\Gamma} x(\tau)+e^{-\frac{\rho}{2} \tau} \eta_{\Gamma}\right) d \tau\right] d s
$$

And the solution of this equation is called a fixed point. According to the theory related to the fixed point method $[7,15]$, the fixed point exists and is unique if there exists a real number $\beta \in(0,1)$, such that for each $t \geq 0$

$$
\|\Psi(x(t))-\Psi(y(t))\| \leq \beta\|x-y\|
$$


which is called the contraction mapping condition. Here, $\|\cdot\|$ is the Frobenius norm [4]. Indeed, we have

$$
\begin{aligned}
\|\Psi(x(t))-\Psi(y(t))\| & =\left\|\int_{0}^{t} e^{\mathcal{A}(t-s)} B R^{-1} B^{T}\left\{\int_{s}^{\infty} e^{-\mathcal{A}^{T}(s-\tau)}\left[Q_{\Gamma}(x(\tau)-y(\tau))\right] d \tau\right\} d s\right\| \\
& \leq \int_{0}^{t}\left\|e^{\mathcal{A}(t-s)} B R^{-1} B^{T}\right\|\left(\int_{s}^{\infty}\left\|e^{-\mathcal{A}^{T}(s-\tau)} Q_{\Gamma}\right\|\|x-y\| d \tau\right) d s \\
& \leq\|x-y\| \times \int_{0}^{t}\left\|e^{\mathcal{A}(t-s)} B R^{-1} B^{T}\right\|\left(\int_{s}^{\infty}\left\|e^{-\mathcal{A}^{T}(s-\tau)} Q_{\Gamma}\right\| d \tau\right) d s \\
& \leq\|x-y\| \times \int_{0}^{t}\left\|e^{\mathcal{A} s} B R^{-1} B^{T}\right\|\left(\int_{0}^{\infty}\left\|e^{\mathcal{A}^{T} \tau} Q_{\Gamma}\right\| d \tau\right) d s \\
& \leq\|x-y\| \times \int_{0}^{\infty}\left\|e^{\mathcal{A} s} B R^{-1} B^{T}\right\|\left(\int_{0}^{\infty}\left\|e^{\mathcal{A}^{T} \tau} Q_{\Gamma}\right\| d \tau\right) d s
\end{aligned}
$$

Let $\beta=\int_{0}^{\infty}\left\|e^{\mathcal{A} s} B R^{-1} B^{T}\right\|\left(\int_{0}^{\infty}\left\|e^{\mathcal{A}^{T} \tau} Q_{\Gamma}\right\| d \tau\right) d s$, we consider Example 2.6.11 and the computational results are based on Matlab.

In Example 2.6.11, we have $A=\left(\begin{array}{cc}1 & -1 \\ 0 & 2\end{array}\right), B=\left(\begin{array}{l}1 \\ 1\end{array}\right), Q=\left(\begin{array}{ll}1 & 0 \\ 0 & 1\end{array}\right), \Gamma=\gamma\left(\begin{array}{cc}1 & 0 \\ 0.5 & 1\end{array}\right), \rho=$ $1, R=1$. When we set $\gamma=2, \beta=12.4298>1$. When we set $\gamma=1, \beta=6.24287>1$. Thus, the fixed point method cannot work in these two situations, while we solve these two situations successfully by our computational method. Actually, if we set $\gamma=0.05$, $\beta=0.894025<1$, which means that we can apply the fixed point method to solve Hamiltonian ODE system (2.64). However, the fixed point method has high computational load since it involves the iteration of function curves.

Our method avoids the contraction mapping condition. It solves the SCE equation system and the only assumption involved is that the corresponding Hamiltonian matrix has no purely imaginary eigenvalues. Also it avoids the iteration of function curves as in the fixed point method. 


\section{Chapter 3}

\section{Mean Field Game Problem}

\subsection{Description of Problem}

Now, we consider a continuous time linear quadratic mean field game (MFG):

$$
\left\{\begin{array}{l}
d x_{i}=\left(A x_{i}+B u_{i}\right) d t+D d W_{i} \\
\min J_{i}\left(u_{i}\right)=E \int_{0}^{\infty} e^{-\rho t}\left(\left|x_{i}-\left(\Gamma x^{(N)}+\eta\right)\right|_{Q}^{2}+u_{i}^{T} R u_{i}\right) d t
\end{array}\right.
$$

The initial states $\left\{x_{i}(0), \quad 1 \leq i \leq N\right\}$ are independent. The noise processes $\left\{W_{i}, \quad 1 \leq\right.$ $i \leq N\}$ are $n_{2}$ dimensional independent standard Brownian motions adapted to $\mathcal{F}_{t}$, which are also independent of $\left\{x_{j}(0), 1 \leq j \leq N\right\}$. The constant matrices $A, B$ and $D$ have compatible dimensions. Given a matrix $Q$, the quadratic form $z^{T} Q z$ may be denoted as $|z|_{Q}^{2}$

According to mean field game theory [9], the decentralized strategies for the above problem can be designed by using the following ODE system:

$$
\left\{\begin{array}{l}
\frac{d \bar{x}}{d t}=\left(A-B R^{-1} B^{T} \Pi\right) \bar{x}-B R^{-1} B^{T} s, \\
\frac{d s}{d t}=Q \Gamma \bar{x}+\left(\rho I-A^{T}+\Pi B R^{-1} B^{T}\right) s+Q \eta .
\end{array}\right.
$$

Letting $\tilde{x}=e^{-\rho t / 2} \bar{x}$ and $\tilde{s}=e^{-\rho t / 2} s$, we have an ODE system of $(\tilde{x}, \tilde{s})$ :

$$
\left\{\begin{array}{l}
\frac{d \tilde{x}}{d t}=\mathcal{A} \tilde{x}-B R^{-1} B^{T} \tilde{s} \\
\frac{d \tilde{s}}{d t}=Q \Gamma \tilde{x}-\mathcal{A}^{T} s+\tilde{\eta}_{\Gamma}
\end{array}\right.
$$

where $\mathcal{A}=A-B R^{-1} B^{T} \Pi-\frac{\rho}{2} I$ and $\tilde{\eta}_{\Gamma}=e^{-\rho t / 2} Q \eta$. 
Notice that $Q \Gamma$ is generally not symmetric, so ODE system (3.3)-(3.4) is not a Hamiltonian ODE system, which means our method cannot be applied directly. However, we can follow the similar idea in Section 2.5 to find a solution $(\bar{x}, s) \in C_{\rho / 2}\left([0, \infty), \mathbb{R}^{2 n}\right)$.

\subsection{Existence of A Solution}

Let

$$
M=\left(\begin{array}{cc}
\mathcal{A} & -B R^{-1} B^{T} \\
Q \Gamma & -\mathcal{A}^{T}
\end{array}\right)
$$

Theorem 3.2.1. If there exists an invertible matrix $U=\left(\begin{array}{cc}U_{11} & U_{12} \\ U_{21} & U_{22}\end{array}\right)$, where $U_{11}$ is inveritble and

$$
U^{-1} M U=\left(\begin{array}{cc}
M_{11} & M_{12} \\
0 & M_{22}
\end{array}\right)
$$

where $M_{11}$ has $n$ stable eigenvalues and $M_{22}$ has $n$ eigenvalues with positive real part. Then we can obtain the following conclusion:

1) For any fixed initial state $x_{0}$ of $O D E$ system (3.3)-(3.4), there exists an initial condition $s_{0}$, such that ODE system (3.3)-(3.4) has a unique solution $(\bar{x}, s) \in C_{\rho / 2}\left([0, \infty), \mathbb{R}^{2 n}\right)$.

2) Such an initial condition $s_{0}$ is unique and is equal to

$$
U_{21} U_{11}^{-1} \bar{x}_{0}+\left(U_{21} U_{11}^{-1} U_{12}-U_{22}\right) \int_{0}^{+\infty} e^{-M_{22} \tau} V_{22} Q \eta e^{-\rho \tau / 2} d \tau
$$

where $V=U^{-1}=\left(\begin{array}{ll}V_{11} & V_{12} \\ V_{21} & V_{22}\end{array}\right)$.

Proof. 1) Proof of existence.

$$
\begin{aligned}
\operatorname{Letting}\left(\begin{array}{l}
\hat{x} \\
\hat{s}
\end{array}\right)=V\left(\begin{array}{l}
\tilde{x} \\
\tilde{s}
\end{array}\right) \text {, the ODE systems of }(\hat{x}, \hat{s}) \text { is: } \\
\qquad \begin{aligned}
\frac{d \hat{x}}{d t} & =M_{11} \hat{x}+M_{12} \hat{s}+V_{12} \tilde{\eta_{\Gamma}}, \\
\frac{d \hat{s}}{d t} & =M_{22} \hat{s}+V_{22} \tilde{\eta_{\Gamma}} .
\end{aligned}
\end{aligned}
$$

Firstly, since $\tilde{\eta}_{\Gamma}=e^{-\rho t / 2} Q \eta$, from (3.8), we can obtain that

$$
\frac{d \hat{s}}{d t}=M_{22} \hat{s}+V_{22} Q \eta e^{-\rho t / 2} .
$$


Thus, the solution of ODE (3.8) has the form:

$$
\hat{s}(t)=e^{M_{22} t} \hat{s}_{0}+\int_{0}^{t} e^{M_{22}(t-\tau)} V_{22} Q \eta e^{-\rho \tau / 2} d \tau .
$$

Since $M_{22}$ is stable, the integral $\int_{0}^{+\infty} e^{-M_{22} \tau} V_{22} Q \eta e^{-\rho \tau / 2} d \tau$ exists and finite. Letting

$$
\hat{s}_{0}=-\int_{0}^{+\infty} e^{-M_{22} \tau} V_{22} Q \eta e^{-\rho \tau / 2} d \tau
$$

we obtain that

$$
\begin{aligned}
\hat{s}(t) & =-\int_{t}^{\infty} e^{M_{22}(t-\tau)} V_{22} Q \eta e^{-\rho \tau / 2} d \tau \\
& =-\int_{0}^{\infty} e^{-M_{22} \tau} V_{22} Q \eta e^{-\rho(\tau+t) / 2} d \tau \\
& =-e^{-\rho t / 2} \int_{0}^{\infty} e^{\left(-M_{22}-\frac{\rho}{2} I\right) \tau} V_{22} Q \eta d \tau .
\end{aligned}
$$

Denote the maximal real part of eigenvalues of $M_{11}$ as $\sigma_{s}$. Since $M_{11}$ is stable, $\sigma_{s}$ is negative. Consider a real positve number $\varepsilon$ such that $\varepsilon<\min \left(-\sigma_{s}, \frac{\rho}{2}\right)$. From equation (3.10), we can obtain

$$
e^{\varepsilon t} \hat{s}(t)=-e^{-\left(\frac{\rho}{2}-\varepsilon\right) t} \int_{0}^{\infty} e^{\left(-M_{22}-\frac{\rho}{2} I\right) \tau} V_{22} Q \eta d \tau
$$

Since $\left(-M_{22}-\frac{\rho}{2} I\right)$ is stable and $\frac{\rho}{2}-\varepsilon>0$, we have that $e^{\varepsilon t} \hat{s}(t)$ is bounded. Thus, $e^{\frac{\rho}{2} t} \hat{s}(t)$ is within $C_{\rho / 2}\left([0, \infty), \mathbb{R}^{n}\right)$.

Secondly, from the ODE (3.7), we can obtain that

$$
\frac{d \hat{x}}{d t}=M_{11} \hat{x}+M_{12} \hat{s}(t)+V_{12} Q \eta e^{-\rho t / 2}
$$

where $\hat{s}(t)$ is the solution in (3.10).

Now consider $e^{\varepsilon t} \hat{x}(t)$, where $\hat{x}(t)$ is the solution of ODE (2.27). We can obtain that

$$
\begin{aligned}
\frac{d\left[e^{\varepsilon t} \hat{x}(t)\right]}{d t} & =\varepsilon e^{\varepsilon t} \hat{x}(t)+e^{\varepsilon t} \frac{d \hat{x}}{d t} \\
& =\varepsilon e^{\varepsilon t} \hat{x}(t)+e^{\varepsilon t}\left[M_{11} \hat{x}(t)+M_{12} \hat{s}(t)+V_{12} Q \eta e^{-\rho t / 2}\right] \\
& =\left(M_{11}+\varepsilon I\right)\left[e^{\varepsilon t} \hat{x}(t)\right]+M_{12}\left[e^{\varepsilon t} \hat{s}(t)\right]+V_{12} Q \eta e^{\left(\varepsilon-\frac{\rho}{2}\right) t} .
\end{aligned}
$$

From (3.11), we can obtain that $\lim _{t \rightarrow+\infty} e^{\varepsilon t} \hat{s}(t)=0$. In addition, since $\left(M_{11}+\varepsilon I\right)$ is stable, for any initial state $\hat{x}_{0}$, we conclude that $e^{\varepsilon t} \hat{x}(t)$ is bounded, which means that $e^{\frac{\rho}{2} t} \hat{x}(t)$ is within $C_{\rho / 2}\left([0, \infty), \mathbb{R}^{n}\right)$ 
Now, we obtain $e^{\frac{\rho}{2} t} \hat{x}(t)$ and $e^{\frac{\rho}{2} t} \hat{s}(t)$ are both within $C_{\rho / 2}\left([0, \infty), \mathbb{R}^{n}\right)$. Since

$$
\left(\begin{array}{l}
\bar{x}(t) \\
s(t)
\end{array}\right)=U\left(\begin{array}{c}
e^{\frac{\rho}{2} t} \hat{x}(t) \\
e^{\frac{\rho}{2} t} \hat{s}(t)
\end{array}\right)
$$

we obtain that $\bar{x}(t) \in C_{\rho / 2}\left([0, \infty), \mathbb{R}^{n}\right)$ and $s(t) \in C_{\rho / 2}\left([0, \infty), \mathbb{R}^{n}\right)$, i.e. $(\bar{x}, s) \in C_{\rho / 2}\left([0, \infty), \mathbb{R}^{2 n}\right)$.

Finally, since

$$
\begin{aligned}
& \bar{x}_{0}=U_{11} \hat{x}_{0}+U_{12} \hat{s}_{0}, \\
& s_{0}=U_{21} \hat{x}_{0}+U_{22} \hat{s}_{0},
\end{aligned}
$$

and $U_{11}$ is invertible, for any fixed $\bar{x}_{0}$ and fixed $\hat{s}_{0}$, there always exists an initial condition

$$
s_{0}=U_{21} U_{11}^{-1}\left(\bar{x}_{0}-U_{12} \hat{s}_{0}\right)+U_{22} \hat{s}_{0} .
$$

Thus, for obtaining $\bar{x} \in C_{\rho / 2}\left([0, \infty), \mathbb{R}^{n}\right)$ and $s \in C_{\rho / 2}\left([0, \infty), \mathbb{R}^{n}\right)$, we can choose the initial condition

$$
\begin{aligned}
s_{0} & =U_{21} U_{11}^{-1}\left(\bar{x}_{0}-U_{12} \hat{s}_{0}\right)+U_{22} \hat{s}_{0} \\
& =U_{21} U_{11}^{-1} \bar{x}_{0}-\left(U_{21} U_{11}^{-1} U_{12}-U_{22}\right) \hat{s}_{0} \\
& =U_{21} U_{11}^{-1} \bar{x}_{0}+\left(U_{21} U_{11}^{-1} U_{12}-U_{22}\right) \int_{0}^{+\infty} e^{-M_{22} \tau} V_{22} Q \eta e^{-\rho \tau / 2} d \tau
\end{aligned}
$$

2) The proof of uniqueness is the same as in the corresponding part of Lemma 2.5.1.

Theorem 3.2.1 provides a method to find a unique solution $(\bar{x}, s) \in C_{\rho / 2}\left([0, \infty), \mathbb{R}^{n}\right)$ when we can find such an invertible matrix $U$. Actually, from the condition of Theorem 2.5.1, this method can be only applied when $M$ has $n$ stable eigenvalues and $n$ eigenvalues with positive real parts. Here we provide an example:

Example 3.2.2. Consider $A=\left(\begin{array}{cc}5 & -5 \\ 0 & 10\end{array}\right), B=\left(\begin{array}{l}1 \\ 1\end{array}\right), Q=\left(\begin{array}{ll}1 & 0 \\ 0 & 1\end{array}\right), \eta=\left(\begin{array}{l}1 \\ 0\end{array}\right), \Gamma=\left(\begin{array}{cc}5 & 0 \\ 2.5 & 5\end{array}\right), \rho=$ $2, R=1$, and the initial state is $x_{0}=\left(\begin{array}{l}1 \\ 1\end{array}\right)$. 


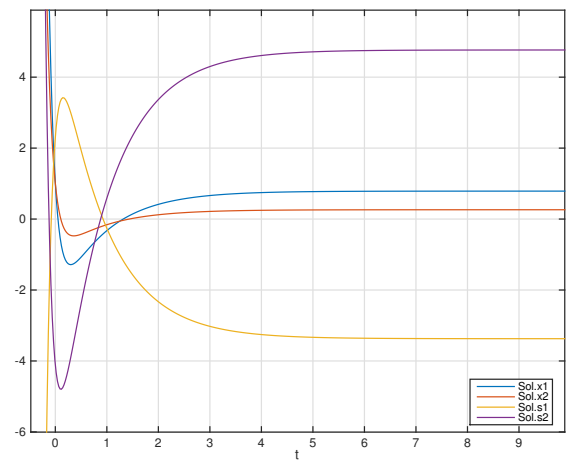

Figure 3.1: Solution of Example 3.2.2

Since

$$
M=\left(\begin{array}{cccc}
14.7999 & -42.0915 & -1.0000 & -1.0000 \\
10.7999 & -28.0915 & -1.0000 & -1.0000 \\
5.0000 & 0 & -14.7999 & -10.7999 \\
2.5000 & 5.0000 & 42.0915 & 28.0915
\end{array}\right),
$$

the eigenvalues of $M$ are $9.2522,1.7783,-2.0950$ and -8.9356 . The Schur decomposition is

$$
U\left(\begin{array}{cccc}
-8.9356 & -13.4806 & 27.2557 & -35.7593 \\
0 & -2.0950 & -46.5898 & -32.5048 \\
0 & 0 & 9.2522 & -15.8037 \\
0 & 0 & 0 & 1.7783
\end{array}\right) U^{-1}
$$

where

$$
U=\left(\begin{array}{cccc}
-0.5425 & -0.6081 & -0.0928 & 0.5721 \\
-0.3060 & -0.4284 & -0.3027 & -0.7945 \\
0.5543 & -0.1947 & -0.7863 & 0.1911 \\
-0.5521 & 0.6394 & -0.5305 & 0.0700
\end{array}\right)
$$

Since $U_{11}=\left(\begin{array}{ll}-0.5425 & -0.6081 \\ -0.3060 & -0.4284\end{array}\right)$ and then $\operatorname{det}\left(U_{11}\right)=0.0464>0$, so $U_{11}$ is invertible. Thus, the initial condition $s_{0}=U_{21} U_{11}^{-1} \bar{x}_{0}+\left(U_{21} U_{11}^{-1} U_{12}-U_{22}\right) \int_{0}^{+\infty} e^{-M_{22} \tau} V_{22} Q \eta e^{-\rho \tau / 2} d \tau=$ $\left(\begin{array}{c}2.31075 \\ -4.11538\end{array}\right)$. From Figure 3.1, it is obvious that the corresponding solution is within $C_{1 / 2}\left([0, \infty), \mathbb{R}^{4}\right)$. 


\section{Chapter 4}

\section{Conclusion}

This thesis develops a methodology to prove the existence and uniqueness of the solutions of the social certainty equivalence equation systems, which is related to social optima in mean field linear-quadratic-Gaussian (LQG) control problems. When the corresponding Hamiltonian matrix has no purely imaginary eigenvalues, a solution within $C_{\rho / 2}\left([0, \infty), \mathbb{R}^{2 n}\right)$ can be found by choosing the initial condition $s_{0}$. On the other hand, this thesis also develops a computational method for the stabilizing solution of continuous-time algebraic Riccati equation by using generalized eigenvectors or Schur vectors. We further extend the stable/unstable subspace decomposition method to solve a linear quadratic mean field game where the ODE system does not have a Hamiltonian structure. 


\section{Bibliography}

[1] G. Albi, Y. P. Choi, M. Fornasier, and D. Kalise. Mean field control hierarchy. arXiv preprint arXiv:1608.01728, 2016.

[2] C. T. Bauch and D. J. Earn. Vaccination and the theory of games. Proceedings of the National Academy of Sciences of the United States of America, 101(36):13391-13394, 2004 .

[3] D. Bauso, R. Pesenti, and M. Tolotti. Opinion dynamics and stubbornness via multi-population mean-field games. Journal of Optimization Theory and Applications, 170(1):266-293, 2016.

[4] J. E. Gentle. Numerical Linear Algebra for Applications in Statistics. Springer Science \& Business Media, 2012.

[5] O. Guéant, J. M. Lasry, and P. L. Lions. Mean Field Games and Applications. Springer Berlin Heidelberg, Berlin, Heidelberg, 2011.

[6] M. Huang, P. E. Caines, and R. P. Malhame. Individual and mass behaviour in large population stochastic wireless power control problems: centralized and Nash equilibrium solutions. In Proceedings of 42nd IEEE Conference on Decision and Control, Maui, HI, USA, pages 98-103, 2003.

[7] M. Huang, P. E. Caines, and R. P. Malhame. Large-population cost-coupled LQG problems with nonuniform agents: Individual-mass behavior and decentralized Nash equilibria. IEEE Transactions on Automatic Control, 52(9):1560-1571, 2007.

[8] M. Huang, P. E. Caines, and R. P. Malhame. Social optima in mean field LQG control: Centralized and decentralized strategies. IEEE Transactions on Automatic Control, 57(7):1736-1751, 2012. 
[9] M. Huang and Y. Ma. Mean field stochastic games with binary action spaces and monotone costs. ArXiv e-prints, January 2017.

[10] M. Huang and S. L. Nguyen. Linear-quadratic mean field teams with a major agent. In Proceedings of 55th IEEE Conference on Decision and Control, Las Vegas, NV, USA, pages 6958-6963, 2016.

[11] P. Lancaster and L. Rodman. Algebraic Riccati Equations. Oxford science publications. Clarendon Press, 1995.

[12] A. Laub. A schur method for solving algebraic Riccati equations. IEEE Transactions on Automatic Control, 24(6):913-921, 1979.

[13] C. Van Loan. A symplectic method for approximating all the eigenvalues of a Hamiltonian matrix. Linear Algebra and its Applications, 61:233 - 251, 1984.

[14] Z. Ma, D. S. Callaway, and I. A. Hiskens. Decentralized charging control of large populations of plug-in electric vehicles. IEEE Transactions on Control Systems Technology, $21(1), 2013$.

[15] M. Nourian, P. E. Caines, R. P. Malhame, and M. Huang. Mean field LQG control in leader-follower stochastic multi-agent systems: Likelihood ratio based adaptation. IEEE Transactions on Automatic Control, 57(11):2801-2816, 2012.

[16] C. Paige and C. Van Loan. A Schur decomposition for Hamiltonian matrices. Linear Algebra and its Applications, 41:11 - 32, 1981.

[17] T. Pappas, A. Laub, and N. Sandell. On the numerical solution of the discrete-time algebraic Riccati equation. IEEE Transactions on Automatic Control, 25(4):631-641, 1980.

[18] Y. Piyapong. Hamiltonian matrices and the algebraic Riccati equation. http://www2.mpi-magdeburg.mpg.de/mpcsc/mitarbeiter/saak/lehre/ Matrixgleichungen/pyuantong_09WS.pdf. 2009.

[19] N. Sen, M. Huang, and R. P. Malhame. Mean field social control with decentralized strategies and optimality characterization. In Proceedings of IEEE 55th Conference on Decision and Control, Las Vegas, NV, USA, pages 6056-6061, 2016. 
[20] L. Smith. Jordan Canonical Form, pages 343-380. Springer New York, New York, NY, 1998.

[21] J. H. Wilkinson. The Algebraic Eigenvalue Problem, volume 87. Clarendon Press Oxford, 1965.

[22] J. Willems. Least squares stationary optimal control and the algebraic Riccati equation. IEEE Transactions on Automatic Control, 16(6):621-634, 1971.

[23] W. M. Wonham. Linear Multivariable Control: A Geometric Approach. Springer, New York, NY, 3rd edition, 2012. 


\section{Appendix A}

\section{Matlab Code}

Here is the matlab code of my example in the section 2.6.2 and 2.6.3.

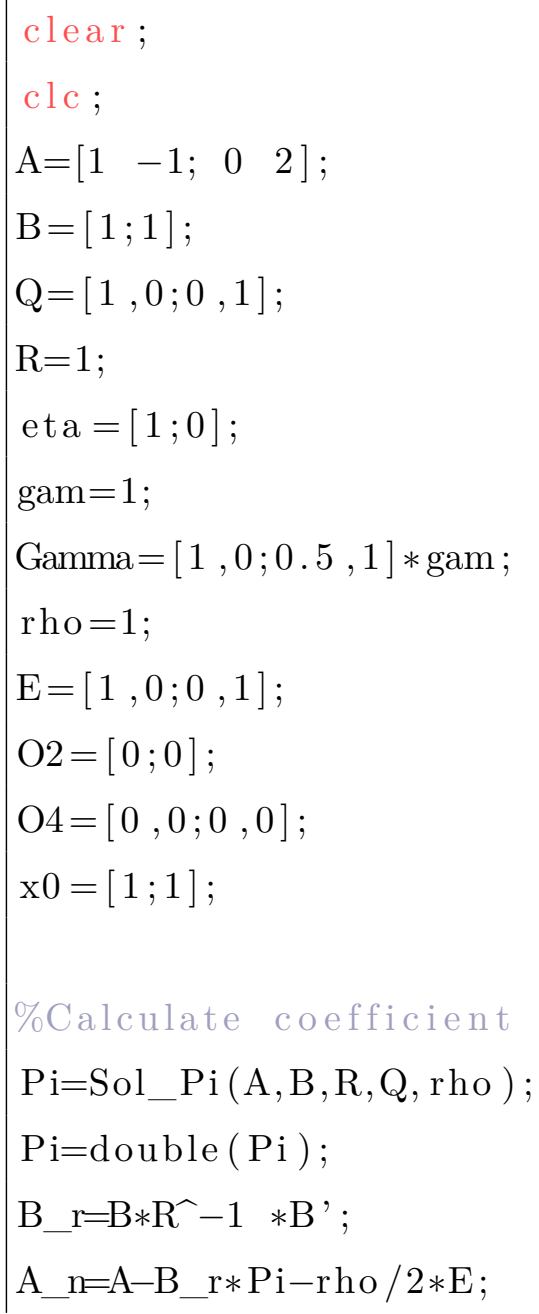




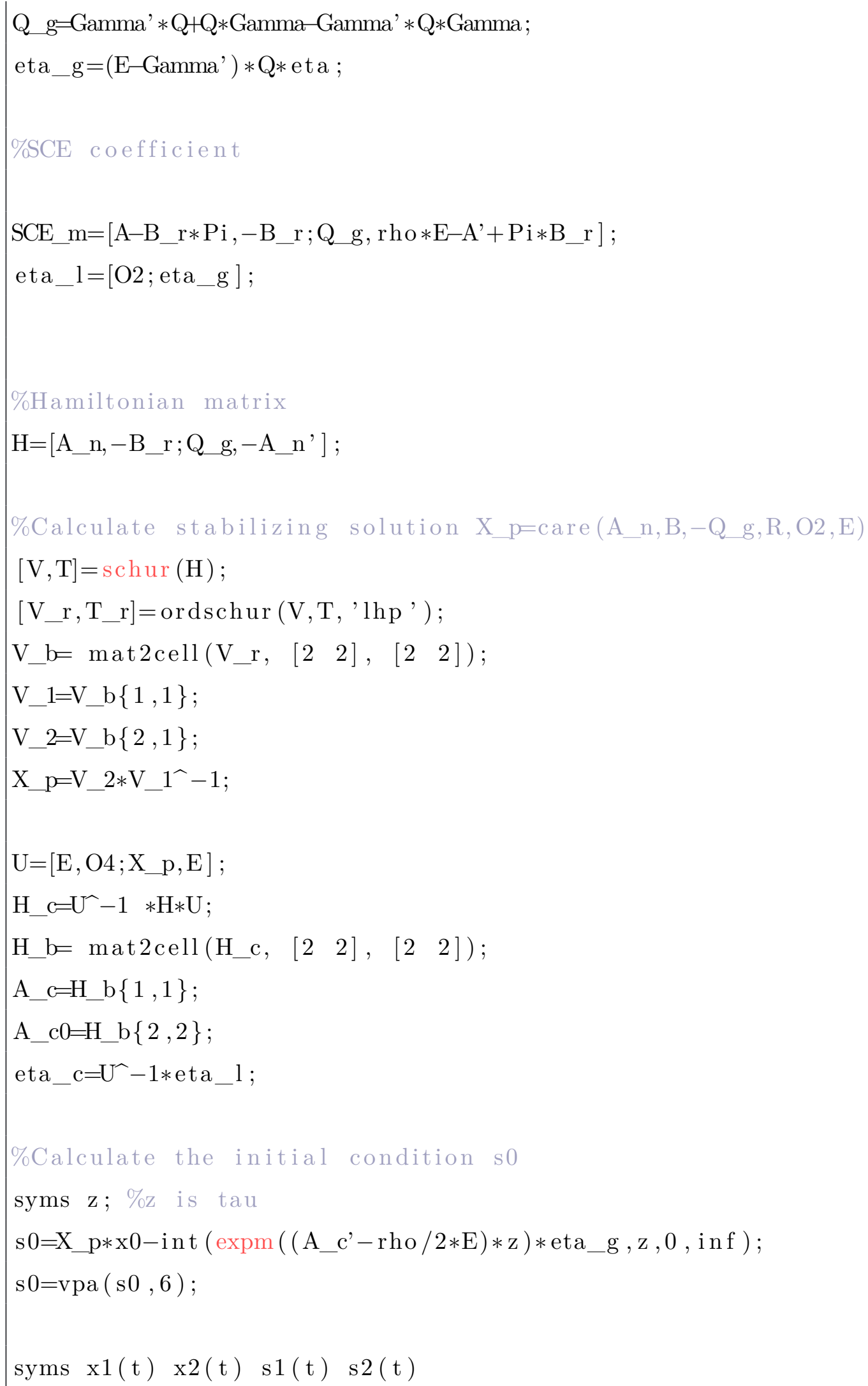




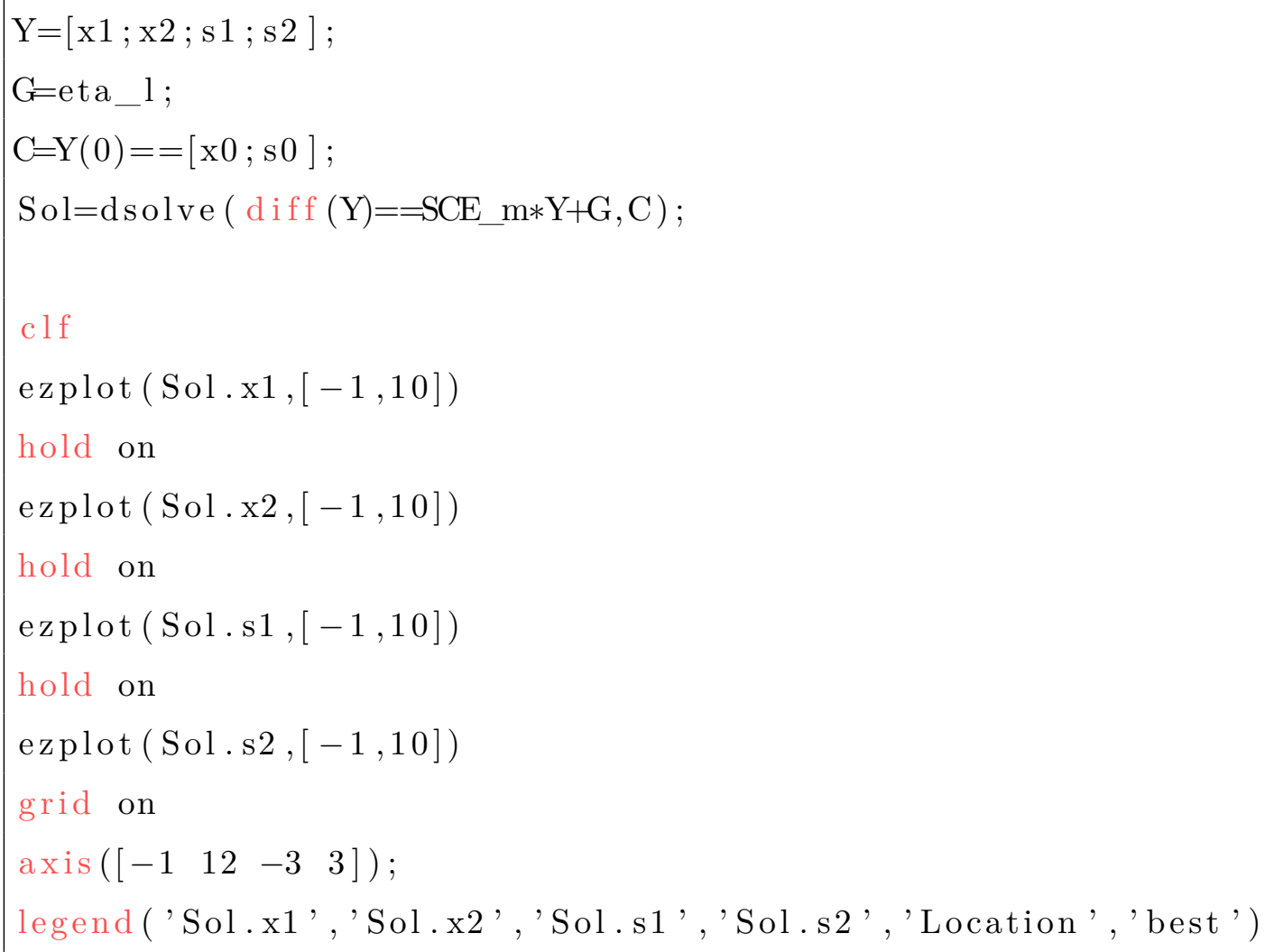




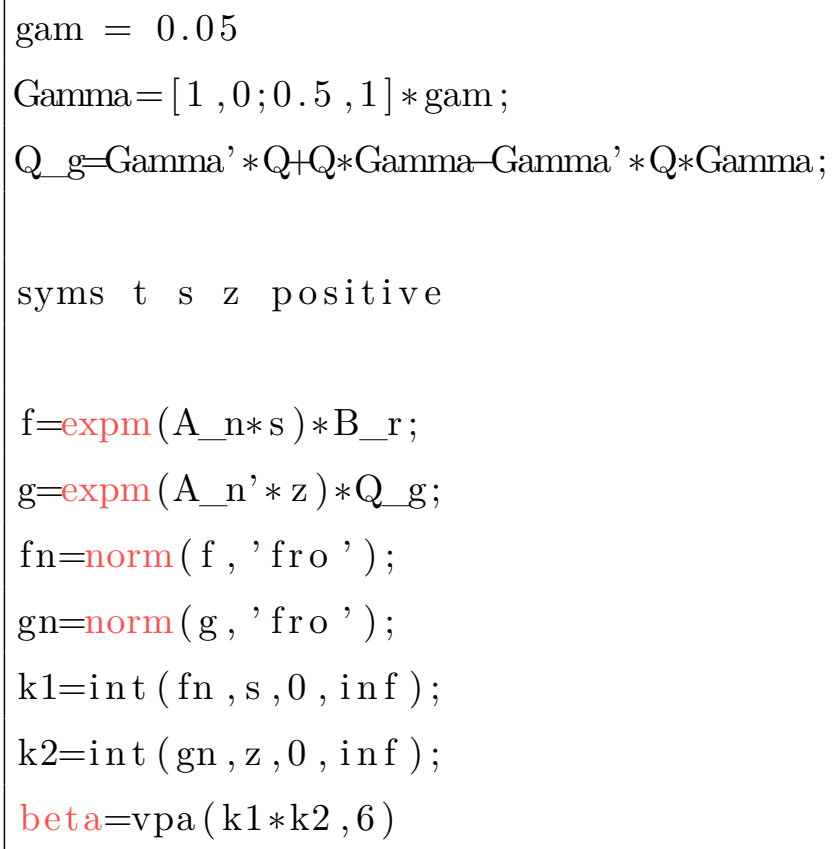

